Article

\title{
Functionalized 1,3-Thiazolidin-4-Ones from 2-Oxo-Acenaphthoquinylidene- and [2.2]Paracyclophanylidene-Thiosemicarbazones
}

\author{
Ashraf A. Aly ${ }^{1, *}$, Nasr K. Mohamed ${ }^{1}$, Alaa A. Hassan ${ }^{1}$, Kamal M. El-Shaieb ${ }^{1}$, \\ Maysa M. Makhlouf ${ }^{1}$ D , Stefan Bräse ${ }^{2,3, *}$, Martin Nieger ${ }^{4}$ and Alan B. Brown ${ }^{5}$ \\ 1 Chemistry Department, Faculty of Science, Minia University, El-Minia 61519, Egypt \\ 2 Institute of Organic Chemistry, Karlsruhe Institute of Technology, Fritz-Haber-Weg 6, \\ 76131 Karlsruhe, Germany \\ 3 Institute of Toxicology and Genetics (ITG) Karlsruhe Institute of Technology (KIT) \\ Hermann-von-Helmholtz-Platz 1, D-76344 Eggenstein-Leopoldshafen, Germany \\ 4 Department of Chemistry, University of Helsinki, P.O. Box 55, A. I. Virtasen aukio I, 00014 Helsinki, Finland \\ 5 Chemistry Program, Florida Institute of Technology, Melbourne, FL 32901, USA \\ * Correspondence: ashrafaly63@yahoo.com (A.A.A.); braese@kit.edu (S.B.)
}

Received: 28 July 2019; Accepted: 17 August 2019; Published: 23 August 2019

check for updates

\begin{abstract}
The reactions of dialkyl acetylenedicarboxylates with various 2-oxo-acenaphthoquinylideneand 4-acetyl[2.2]paracyclophanylidene-thiosemicarbazones were investigated. Using simple experimental procedures, 1,3-Thiazolidin-4-ones derived from acenaphthequinone or [2.2]paracyclophane were obtained as major products in good yields. In the case of allyl derivative of acenaphthoquinylidene-thiosemicarbazones, a complex structure of tetramethyl 5-(2-((Z,E)-N-allyl-N'-(2-oxoacenaphthylen-1(2H)-ylidene)carbamohydrazonoyl)thio)-1,2,3-tris(methoxycarbonyl)-cyclopropyl)-4-methoxy-7-oxabicyclo[2.2.1]hepta-2,5-diene-1,2,3,6-tetracarboxylate was formed. Single crystal X-ray analysis was used as an efficient tool to confirm the structure of the synthesized compounds as well as different spectroscopic data $\left({ }^{1} \mathrm{H}-\mathrm{NMR},{ }^{13} \mathrm{C}-\mathrm{NMR}, 2 \mathrm{D}-\mathrm{NMR}\right.$, mass spectrometry and elemental analysis). The mechanism of the obtained products was discussed.
\end{abstract}

Keywords: acenaphthequinone; 4-acetyl[2.2]paracyclophane; thiosemicarbazone; dialkylacetylenedicarboxylates; 1,3-thiazolidinones; DMAD tetramer

\section{Introduction}

Of interest due to their high biological activities, 1,3-Thiazolidin-4-one derivatives are very important core structures of many heterocyclic compounds. Compounds with a thiazolidinone structure have been reported as anti-inflammatory and analgesic [1], antioxidants [2], antitubercular [1,3], antimicrobial [2,4] and antifungal [4], antiviral (especially as anti-HIV agents [5]), anticancer [1], anticonvulsants [6], and antidiabetic agents [6,7]. Recently, various synthetic routes were used for the synthesis of different thiazolidinones from several thiosemicarbazides and thiocarbohydrazides by using different reagents, and their biological evaluation was also discussed [8,9]. Thiosemicarbazones were used as a starting material for the synthesis of different heterocyclic compounds, i.e., naphtho-thiazole and naphtho-thiadiazepines [10], 4-thiazolidinone derivatives [11,12], 1,3,4-thiadiazoles [13] which possess high biological activities, e.g., anticancer [14], antioxidants [15], antifungal [16], and antibacterial [17].

Acenaphthequinone (acenaphthylene-1,2-dione) is a quinone derivative, widely used as significant starting material for the synthesis of various heterocycles, and as a key intermediate in organic synthesis for different reactions and pharmaceutical applications [18-21]. Some derivatives of acenaphthequinone 
have been used as biological active compounds, dyes, pharmaceuticals, drugs, pesticides, and therapeutic agents [22-24]. Acenaphthylene-1,2-dione is one of the important reagents for constructing new heterocycles, e.g., spirooxadiazoline [25], spiroacenaphthyl-3,4'-pyranopyrazoles [26], fused acenaphthopyrrolizines and spiroacenaphthene pyrrole [27], dispiropyrrolidines [28,29], and polycyclic heterocycles $[29,30]$ via reactions such as the Huisgen reaction, 1,3-dipolar cycloaddition, and [3+2]cycloaddition reactions. One pot synthesis of 2-oxo-1,2-dihydroacenaphthylphosphonates was obtained by using a Knoevenagel-phospha-Michael reaction of acenaphthequinone, malononitrile, and diethylphosphite catalyzed by a 1,4-diazabicyclo-[2.2.2]octane (DABCO)-based ionic liquid [31].

Recently, three component reactions were widely used as one of the most efficient routes for the synthesis of many heterocyclic rings. Consequently, three component reactions of acenaphthequinone, proline derivatives and dialkyl acetylenedicarboxylates gave azocinobenzoisoquinolone-dicarboxylates through [3+2]cycloaddition of azomethine ylides followed by ring expansion [32]. In addition, the domino three component reaction of isoquinolinium salts, acenaphthequinone and indanedione in basic media yielded 2 -acenaphthylidenespiro[indane-2,1'-pyrrolo[2,1-a] isoquinolones] [33]. Using a similar synthetic strategy, bis-spiro-oxoacenaphthalenes were obtained via the reaction of acenaphthalene-1,2-dione, active alkynes, and 4-cyanopyridine in 1,2-dimethoxyethane (DME) at room temperature [34]. The reaction of dialkyl acetylenedicarboxylate with acenaphthalenedione and $\mathrm{CS}_{2}$ in t-butylphosphine afforded oxoindolin-3-ylidene-1,3-dithioles [35]. Also, spirodihydropyridine derivatives were obtained in high yields from the reaction of acenaphthylidene malononitrile and Zwitter ionic intermediate (generated from primary amine and dimethyl acetylenedicarboxylate (DMAD) [36].

Since the maturation of the synthetic methodologies, with the possibilities for the fine-tuning of structural and functional properties, [2.2]paracyclophane (PC) chemistry has evolved-from functional molecules to functional materials [37] - and is rapidly growing from a structural and synthetic curiosity to a prevalent scaffold in asymmetric synthesis [37], $\pi$-stacked polymers, energy materials, and functional perylene coatings that find broad applications in bio-material science and as natural products [38-40]. Aly et al. prepared various heterocycles derived from [2.2]paracyclophane such as a five-membered ring (i.e., imidazolone and pyrrole [41-44] and six-membered ring (i.e., pyridine [45]). Additionally, we reported on the synthesis of various paracyclophanyl-substituted thiosemicarbazones, thiocarbazones and thioureas then studied their complexation towards tridentate and bidentate copper complexes [46]. Inspired by the aforementioned, we aim here to synthesize various thiazolidinone derivatives of both acenaphthequinone and [2.2]paracyclophane.

\section{Results and Discussion}

Acenaphthequinones conjugated with $\mathrm{NH}, \mathrm{SH}, \mathrm{CH}=\mathrm{N}$ and $\mathrm{NH}_{2}$ groups are of considerable interest in organic synthesis and were produced from the condensation of thiosemicarbazide and 4-substituted thiosemicarbazides with acenaphthylene-1,2-dione [47]. A literature survey revealed, that acenaphthequinone (1) was reacted with thiosemicarbazide $2 \mathbf{a}$ either in ethanol at room temperature and catalyzed by acidic ionic liquid furnished spiro-1,2,4-triazolidine-3-thiones 3a [48] or in refluxing chloroform in the presence of glacial acetic acid $(\mathrm{AcOH})$ yielded acenaphtho[1,2-e]-1,2,4-triazine-9(8H)-thione 4 (Scheme 1) [49]. 


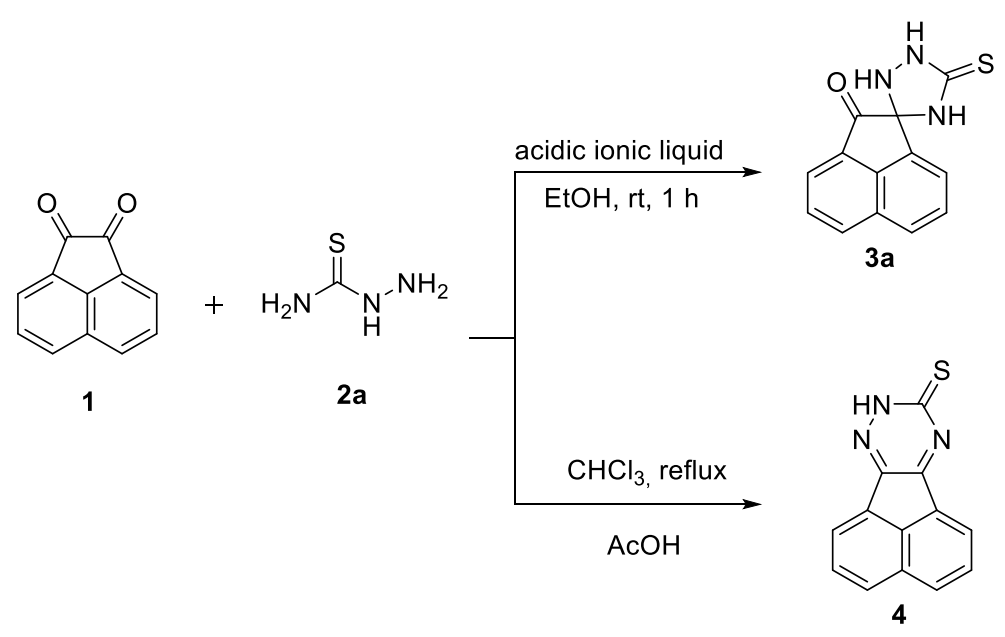

Scheme 1. Previous work on acenaphthalene-1,2-dione 1 with thiosemicarbazide 2 a.

\subsection{Preparation of Compounds 5a-e}

It was obvious that the reaction conditions, e.g., solvent, temperature and/or catalyst play a vital role on the reaction efficiency and product obtained. Various conditions were investigated and the most efficient method was the condensation of thiosemicarbazide derivatives with acenaphthalene-1,2-dione in refluxing ethanol in the presence of $\mathrm{Et}_{3} \mathrm{~N}$ to give (Z)-N-unsubstituted/substituted-2-(2-oxoacenaphthylen-1(2H)-ylidene)hydrazinecarbothioamide derivatives (Scheme 2) instead of the aforementioned spiro-1,2,4-triazolidine-3-thione (3a) [48] and 1,2,4-triazine-3-thione 4 [49]. Although (Z)-2-(2-oxoacenaphthylen-1(2H)-ylidene) hydrazinecarbothioamide $5 \mathbf{a}$ was previously reported [50], its structure was confirmed herein using single crystal X-ray analysis (Figure 1).<smiles>O=C1C(=O)c2cccc3cccc1c23</smiles>

1<smiles>[R]NC(=S)NN</smiles>

2a-e<smiles>[R]NC(=S)N/N=C1\C(=O)c2cccc3cccc1c23</smiles>

5a-e $(70-80 \%)$

2 and 5a-e: $\mathbf{a}, \mathrm{R}=\mathrm{H} ; \mathbf{b}, \mathrm{R}=\mathrm{Ph} ; \mathbf{c}$, cyclohexyl; d, allyl, e, ethyl.

$5 a(70 \%), 5 b(80 \%), 5 c(75 \%), 5 d(74 \%), 5 e(72 \%)$

Scheme 2. Reactions of acenaphthoqinone 1 with thiosemicarbazides 2 a-e. 


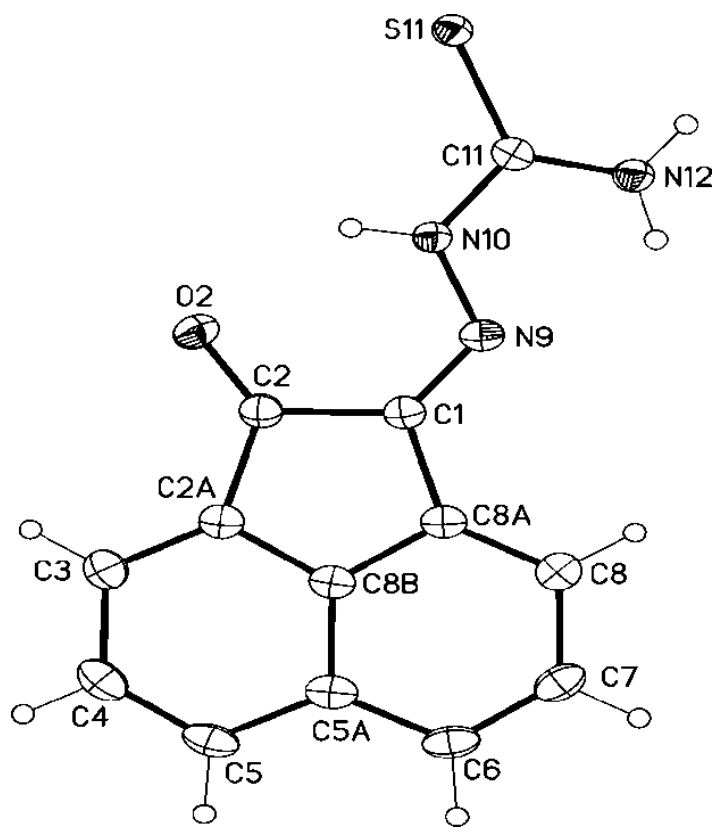

Figure 1. Molecular structure of one of the two crystallographic independent molecules of 5a (displacement parameters are drawn at $50 \%$ probability level).

\subsection{Reaction of Compounds 5a-e with Dimethyl Acetylenedicarboxylate $(D M A D, \mathbf{6})$}

A mixture of dimethyl acetylenedicarboxylate (DMAD, 6a) and (Z)-N-unsubstituted/ substituted-2-(2-oxoacenaphthylen-1-(2H)-ylidene)thiosemicarbazones $5 \mathbf{a}-\mathbf{e}$, was refluxed in absolute EtOH for 20-30 min. The reaction revealed the complete disappearance of compounds $5 \mathbf{a}-\mathbf{e}$ and $\mathbf{6 a}$ to give compounds $\mathbf{7 a - e}$ in $70-76 \%$ yields (Scheme 3). Surprisingly, only in the case of allyl derivative $\mathbf{5 d}$, an unexpected compound $\mathbf{8 d}$ was obtained in $10 \%$ yield (Scheme 3 ). Different spectroscopic data were insufficient to determine completely the correct structure of this compound.

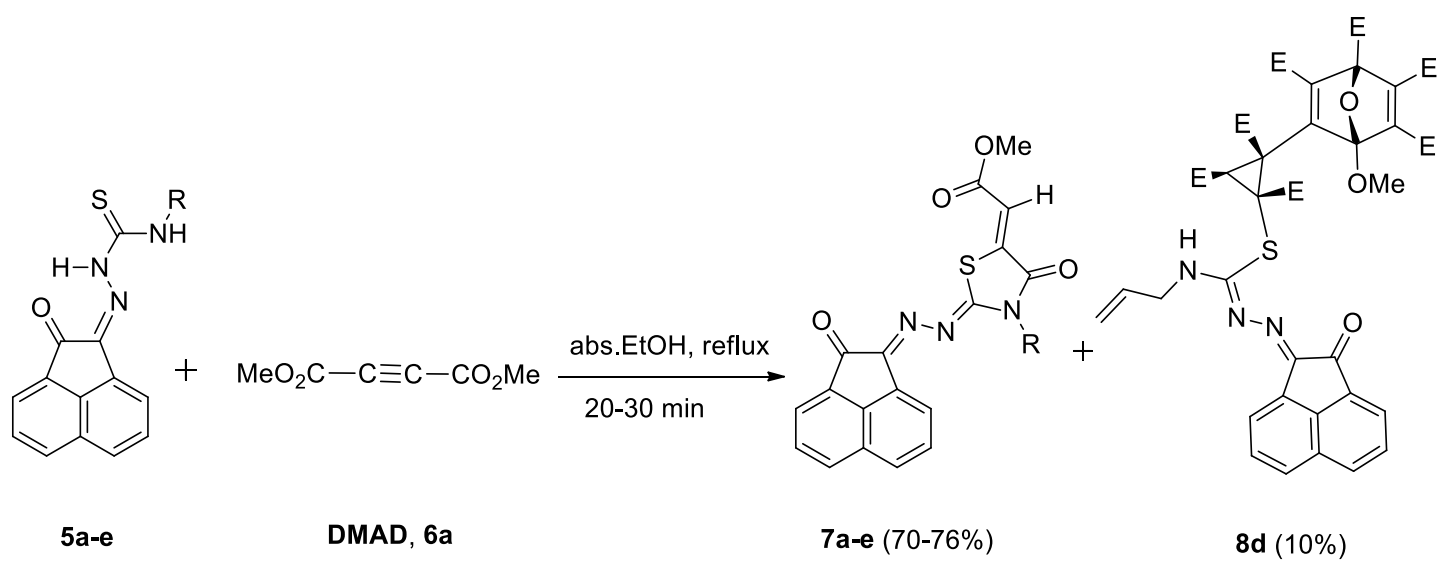

5a-e, 7a-e: a, R = H; b, R = Ph; c, cyclohexyl; d, allyl, e, ethyl.

Scheme 3. Reaction of 2-oxo-acenapthoquinylidene-thiosemicarbazones 5a-e with DMAD, $\mathbf{6 a}$.

Different nucleophilic sites on thiosemicarbazone derivatives $\mathbf{5 a}-\mathbf{e}$ were expected to participate in the reaction and formation of the product. Nucleophilic attack of lone pair of electrons of the sulfur atom in the thione group in 5a-e to the acetylenic carbon in 6a would give the Zwitter ions 9a-e. Neutralization of $\mathbf{9 a - e}$ would give the intermediate 10a-e. Subsequently another nucleophilic attack from a lone pair of electrons of ${ }^{2} \mathrm{NH}$ or ${ }^{4} \mathrm{NH}$ on $\mathrm{CO}_{2} \mathrm{Me}$; would yield the corresponding 
4-thiazolidinones 7a-e and/or 12a-e via the loss of methanol (Scheme 4). The ${ }^{1} \mathrm{H}$ and ${ }^{13} \mathrm{C}-\mathrm{NMR}$ spectral data (see details in Supplementary Materials) were used as an important tool for excluding some alternative structures. Furthermore, in the case of 7a-e and 12a-e, X-ray analyses were used as a useful tool to determine the correct structure and to prove that the 1,3-thiazolidine-4-ones 7a-e were formed rather than $12 \mathrm{a}-\mathbf{e}$.
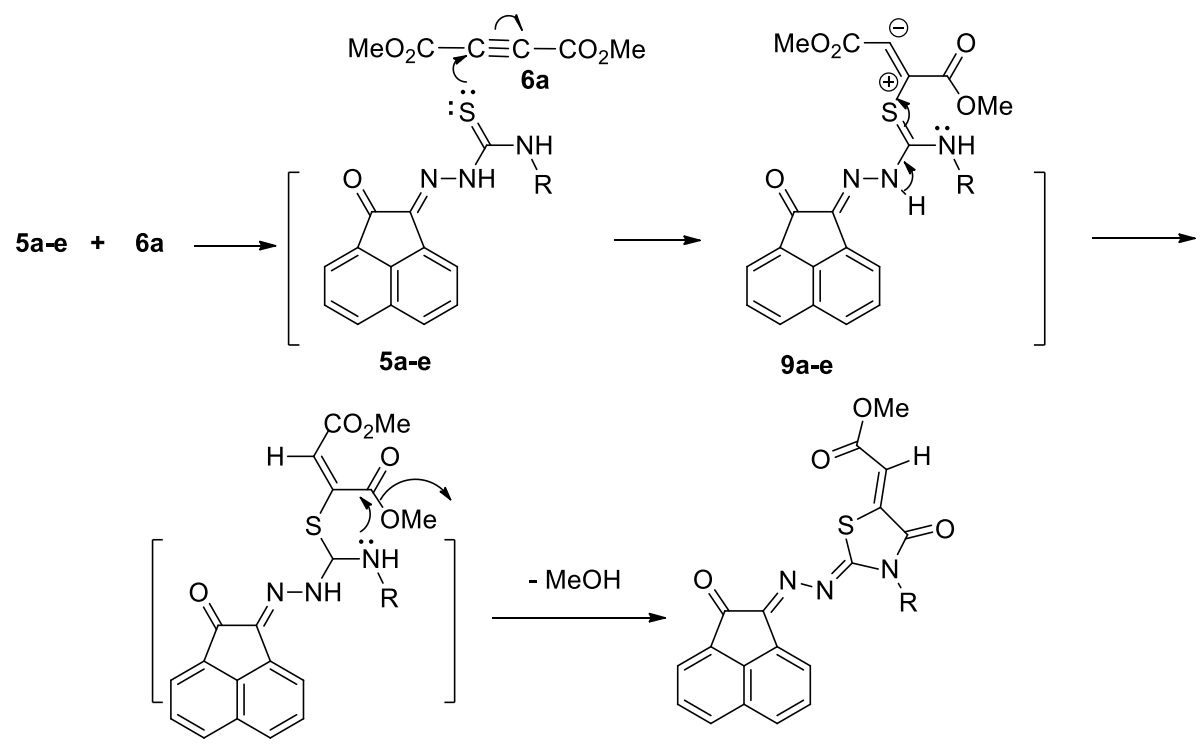

10a-e

7a-e

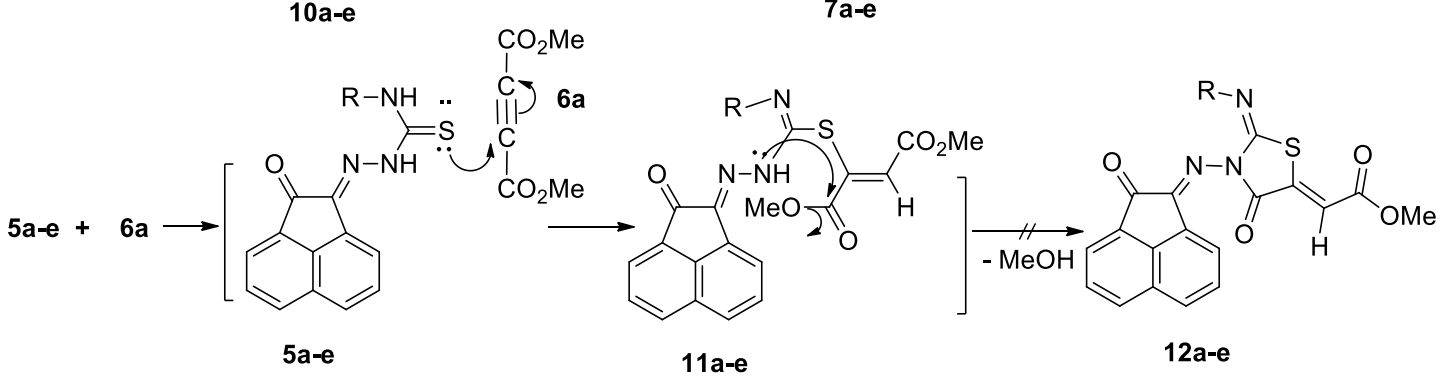

5, 7, 9-12a-e; a; R = H, b; Ph, c; cyclohexyl, d; allyl, e; Et

Scheme 4. The mechanism describes the formation of compounds $7 \mathbf{a}-\mathbf{e}$ and 12a-e.

For example, the IR spectrum of $7 \mathbf{a}$ showed absorption bands at $v=3489 \mathrm{~cm}^{-1}$ for the $\mathrm{NH}$ group, $1715,1696 \mathrm{~cm}^{-1}$ for $\mathrm{C}=\mathrm{O}$ as well as 1623 and $1609 \mathrm{~cm}^{-1}$ for $\mathrm{C}=\mathrm{N}$ and $\mathrm{Ar}-\mathrm{C}=\mathrm{C}$ groups. The ${ }^{1} \mathrm{H}-\mathrm{NMR}$ spectrum of $7 \mathrm{a}$ clearly proved that there are two singlets one at $\delta=3.81 \mathrm{ppm}$ with $3 \mathrm{H}$ integral corresponding to one methoxy group, the other at $\delta=6.73 \mathrm{ppm}$ with $1 \mathrm{H}$ integral relating to vinyl- $\mathrm{CH}$ group. Acenaphthequinone protons resonated at $\delta=7.85-8.35 \mathrm{ppm}$ due to six aromatic protons. A broad NH group also resonated as a singlet at $13.46 \mathrm{ppm}$. Further, ${ }^{13} \mathrm{C}-\mathrm{NMR}$ of 7 a showed further signals at $\delta=52.49,115.27,142.07$, (151.66 and 154.23), 165.78, 166.01 and 183.77 corresponding to $\mathrm{OCH}_{3},-\mathrm{CH}=$, C5-thiazole, (C2-thiazole and $\mathrm{C}=\mathrm{N}$ ), cyclic- $\mathrm{C}=\mathrm{O}, \mathrm{CO}_{2} \mathrm{Me}, \mathrm{C}=\mathrm{O}$. The 2D-NMR further exhibited different correlations between $\mathrm{C}, \mathrm{H}, \mathrm{N}$ (Table 1 and Figure 2). The elemental analysis of $7 \mathbf{a}$ suggests that it has $\mathrm{C}_{18} \mathrm{H}_{11} \mathrm{~N}_{3} \mathrm{O}_{4} \mathrm{~S}$ formula. This was further investigated from mass spectrum giving a molecular ion at $m / z=365(10 \%)$. 
Table 1. NMR spectroscopic data of 7a.

\begin{tabular}{|c|c|c|c|}
\hline${ }^{1} \mathrm{H}-\mathrm{NMR}:$ & \multicolumn{2}{|c|}{${ }^{1} \mathrm{H}_{-}{ }^{1} \mathrm{H}$ COSY: } & Assignment: \\
\hline $13.46(\mathrm{~b} ; 1 \mathrm{H})$ & & & NH-3' \\
\hline $8.35(\mathrm{~d}, J=8.2 ; 1 \mathrm{H})$ & \multicolumn{2}{|r|}{7.86} & $\mathrm{H}-5$ \\
\hline $8.20(\mathrm{~d}, J=8.2 ; 1 \mathrm{H})$ & \multicolumn{2}{|r|}{7.85} & H-6 \\
\hline $8.01(\mathrm{~d}, J=7.8 ; 1 \mathrm{H})$ & \multicolumn{2}{|r|}{7.86} & $\mathrm{H}-3$ \\
\hline $7.99(\mathrm{~d}, J=7.0 ; 1 \mathrm{H})$ & \multicolumn{2}{|r|}{7.85} & $\mathrm{H}-8$ \\
\hline $7.86(“ \mathrm{t}$ ", $J=7.9 ; 1 \mathrm{H}) 8.35,8.01$ & \multicolumn{2}{|r|}{$\mathrm{H}-4$} & \\
\hline $7.85(" \mathrm{t} t$ ", $J=8.0 ; 1 \mathrm{H})$ & \multirow{3}{*}{\multicolumn{2}{|c|}{$8.20,7.99$}} & $\mathrm{H}-7$ \\
\hline $6.73(\mathrm{~s} ; 1 \mathrm{H})$ & & & $\mathrm{H}-5 \mathrm{a}^{\prime}$ \\
\hline $3.81(\mathrm{~s} ; 3 \mathrm{H})$ & & & $\mathrm{H}-5 \mathrm{c}^{\prime}$ \\
\hline${ }^{13}$ C NMR: & \multicolumn{2}{|c|}{${ }^{1} \mathrm{H}-{ }^{13} \mathrm{C}$ HSQC: $\mathrm{H}^{13} \mathrm{C}$ HMBC: } & Assignment: \\
\hline 183.77 & \multirow{2}{*}{\multicolumn{2}{|c|}{$8.01,7.86$}} & $\mathrm{C}-2$ \\
\hline 166.01 & & & C-4 \\
\hline 165.78 & \multirow{2}{*}{\multicolumn{2}{|c|}{$6.73,3.81$}} & $C-5^{\prime}$ \\
\hline 154.23 & & & $C-2^{\prime}$ \\
\hline 151.66 & \multirow{5}{*}{8.35} & 7.99 & $\mathrm{C}-1$ \\
\hline 142.07 & & 6.73 & $C-5^{\prime}$ \\
\hline 139.71 & & $8.35,8.20,8.01,7.86,7.85$ & C-9a \\
\hline 131.86 & & $8.20,8.01,7.86$ & $C-5$ \\
\hline $131.15,131.05,130.06$ & & $8.35,8.20,8.01,7.86,7.85$ & $C-2 a, 5 a, 9$ \\
\hline $128.95,128.59$ & $7.86,7.85$ & $8.35,7.99$ & C- $-4,7$ \\
\hline 128.20 & 8.20 & 8.20 & C-6 \\
\hline 121.52 & 8.01 & 8.35 & $C-3$ \\
\hline 118.82 & 7.99 & 8.20 & C-8 \\
\hline 115.27 & 6.73 & $6.73,3.81$ & $C-5 a^{\prime}$ \\
\hline 52.49 & 3.81 & 3.81 & $C-5 c^{\prime}$ \\
\hline
\end{tabular}

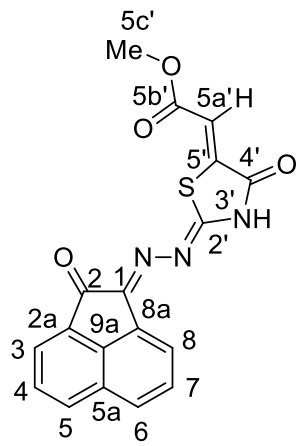

Figure 2. The distinctive carbons in compound 7a.

Additionally, the structure of (Z)-methyl 2-((Z)-3-allyl-4-oxo-2-((E)-(2-oxoacenaphthylen-1(2H)ylidene)hydrazono)thiazolidin-5-ylidene)acetate (7d) has been unambiguously confirmed via a single-crystal X-ray structure analysis (Figure 3). The characteristic properties of compound $\mathbf{7 d}$ are that the C2-N13 and C15-N14, double bonds (note that the crystallographic numbering does not correspond to the systematic IUPAC numbering rules) exhibit bond lengths of 1.2912 (16) $\AA$ and 1.2892 (16) $\AA$, respectively, that are a little shorter than $\mathrm{C}=\mathrm{N} \delta$ bonds due to high resonance in $7 \mathrm{~d}$. The dihedral angle of N19-C15-N14-N13 is $178.82(9)^{\circ}$, showing that it has a trans configuration relating to N19. Bond lengths C1-C2 and N13-N14 are 1.5345 (16) and 1.3950 (14), respectively, which are shorter than the corresponding $\mathrm{C}-\mathrm{C}$ and N-N single bonds due to high resonance in this compound. From $\mathrm{X}$-ray analysis, it was also observed that the thiazolidine $\mathrm{C}=\mathrm{O}$ has a cisoid geometry regarding to vinyl $\mathrm{CH}$ [torsion angle O18-C18-C17-C23-2.2 (2) ${ }^{\circ}$. 


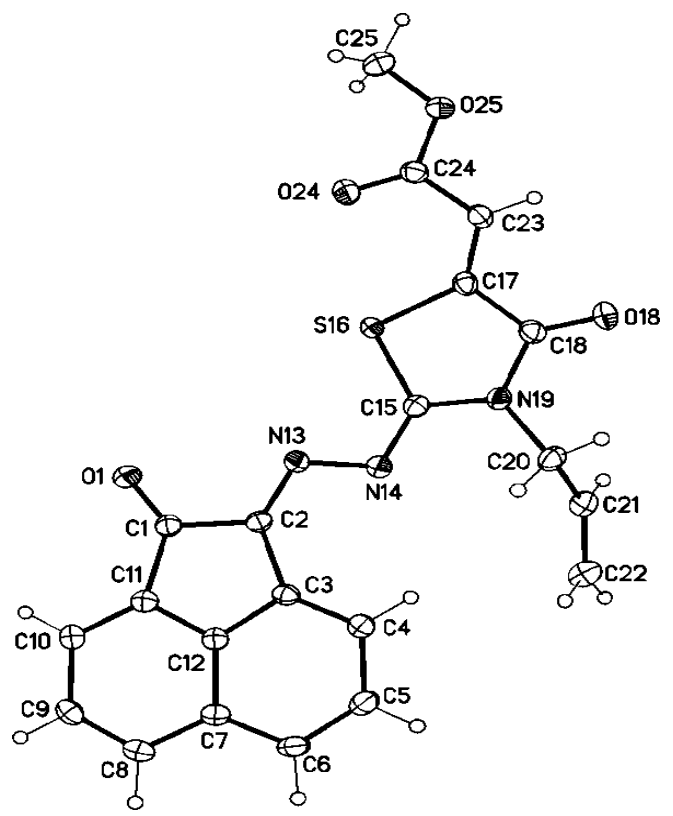

Figure 3. Molecular structure of $\mathbf{7 d}$ (displacement parameters are drawn at 50\% probability level).

A literature survey revealed that, the tetramerization reaction is one of the most fascinating reactions of both dialkyl acetylenedicarboxylic acid derivatives occurring at $25^{\circ} \mathrm{C}$ for several days or by heating it alone or in solution for several hours [51-53]. Furthermore, trimer [54] and dimer [55] forms were reported for compound 6a. Recently, Huang et al. [56] have reported the reaction of $2,2^{\prime}$-bis(azaphosphindole) with four equivalents of $\mathbf{6 a}$ in THF at room temperature to afford a tetramer complex of 6 a with $2,2^{\prime}$-bis(azaphosphindole).

The structure of $\mathbf{8 d}$ was resolved by using single crystal X-ray analysis and it was found that it is tetramethyl 5-(2-(( $Z, E)-N$-allyl- $N^{\prime}$-(2-oxoacenaphthylen-1(2H)-ylidene)carbamo-hydrazonoyl) -thio)-1,2,3-tris(methoxycarbonyl)cyclopropyl)-4-methoxy-7-oxabicyclo-[2.2.1]hepta-2,5-diene-1,2,3,6tetracarboxylate (8d) (Figure 4). To our best knowledge, it would be the first X-ray structure of that tetramer of $\mathbf{6 a}$. It is interesting to note that only one diastereomer was isolated (at least being isolated).

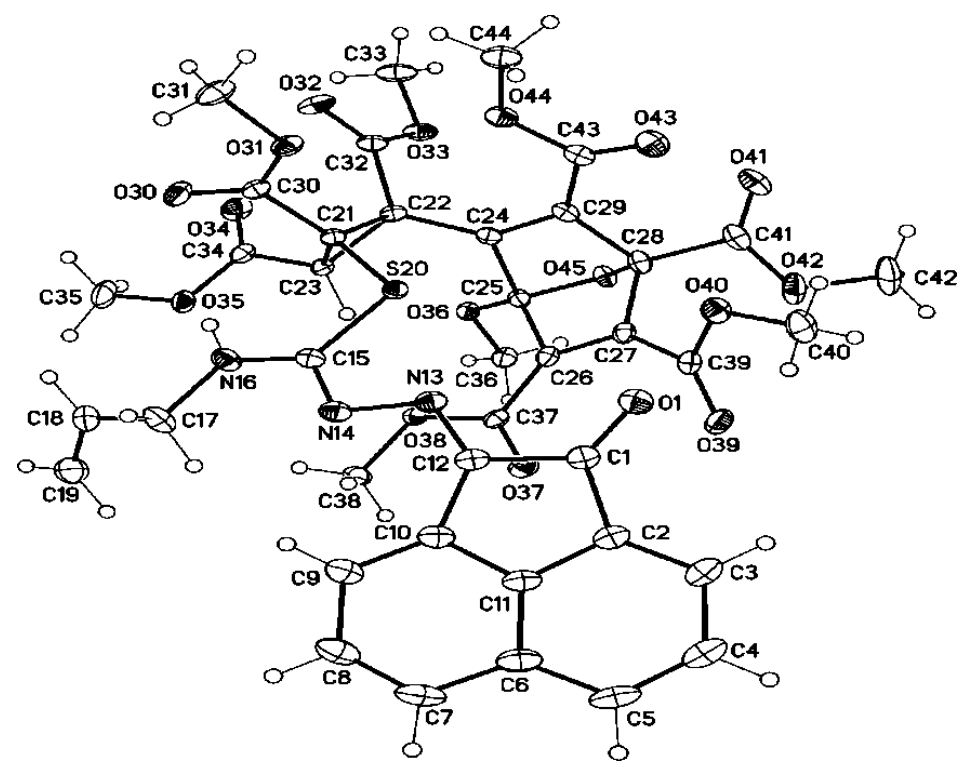

Figure 4. Molecular structure of one of the two crystallographic independent molecules of $\mathbf{8 d}$ (displacement parameters are drawn at $30 \%$ probability level). 
The unusual reactivity of $\mathbf{6}$ a towards allyl derivative of thiosemicarbazones $\mathbf{5} \mathbf{d}$ obviously involves four molecules of 6 reacting with one molecule of 5d. The mechanism presumably begins via dimerization of $\mathbf{6 a}$ (acting as dienophile and as 1,3-dipole) to afford $\mathbf{1 3}$, which then rearranged to give 14. Addition of the third molecule of 6 a to the intermediate 14 would give the intermediate 15 , which would be neutralized to give cyclopropene 16 (Scheme 5). Diels-Alder reaction of 16 and the fourth molecule of 6a, yield the complex 17 [56] (Scheme 5). Subsequently, a nucleophilic attack of thione-lone pair of thiosemicarbazone $\mathbf{5} \mathbf{d}$ to the double bond of the cyclopropene ring would give the Zwitter ion $\mathbf{1 8}$, which would be neutralized to give compound $\mathbf{8 d}$ (Scheme 5).

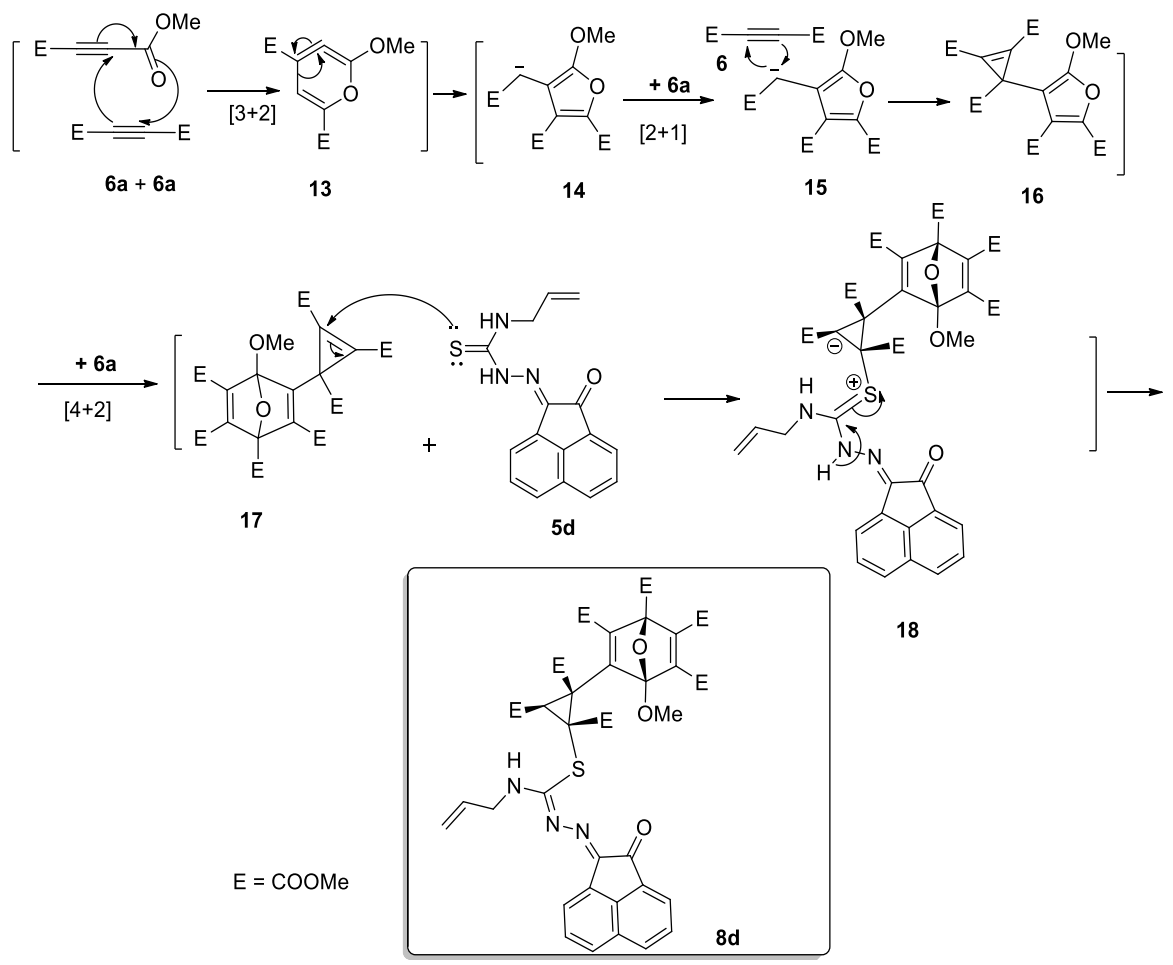

Scheme 5. Plausible mechanism for the synthesis of compound $\mathbf{8 d}$.

The formation of a complex structure may be further used as a Supplementary tool for the formation of 1,3-thiazolidinone derivative $\mathbf{7 d}$, which might be attributed to the instability of $\mathbf{8 d}$ during the course of a reaction. It can be also suggested that another nucleophilic attack of NH lone-pair of compound $\mathbf{8 d}$ to one of the carbonyl ester group of the cyclopropane ring afforded the intermediate 19 that would be rearranged giving the 4-thiazolidinone 7d and $\mathbf{2 0}$ (Scheme 6). However, compound 20 was unfortunately not isolated.

On the other hand, the synthesis of 4-acetyl[2.2]paracyclophanylidene thiosemicarbazone (22a) was established from the reaction between 4-acetyl[2.2]paracyclophane (21) and thiosemicarbazide (2a) and by applying the procedure described by Aly et al. [46].

Similarly, the reaction of $\mathbf{2 1}$ with $\mathbf{2 f}$ gave $\mathbf{2 2 f}$ in $60 \%$ yield (Scheme 7). If two equivalents of $\mathbf{2 1}$ were reacted with one equivalent of thiocarbohydrazide (2f), the reaction gave bis-4-acetyl-[2.2]paracyclophanylidene-hydrazine-1-carbothiohydrazone (23) in 88\% yield. In addition, refluxing of the two equivalents of $N$-cyclohexylhydrazinecarbothioamide (2c) with 4,15-diformyl-2.2]paracyclophane (24) yielded the desired 2,2'-(1,4(1,4)-dibenzenacyclohexaphane- $1^{2}, 4^{3}$-diylbis(methane-ylylidene))bis-( $N$-cyclohexylhydrazine-1-carbothioamide (25) in $80 \%$ yield (Scheme 7). The X-ray structure analysis of compound 25 is as shown in Figure 5. 


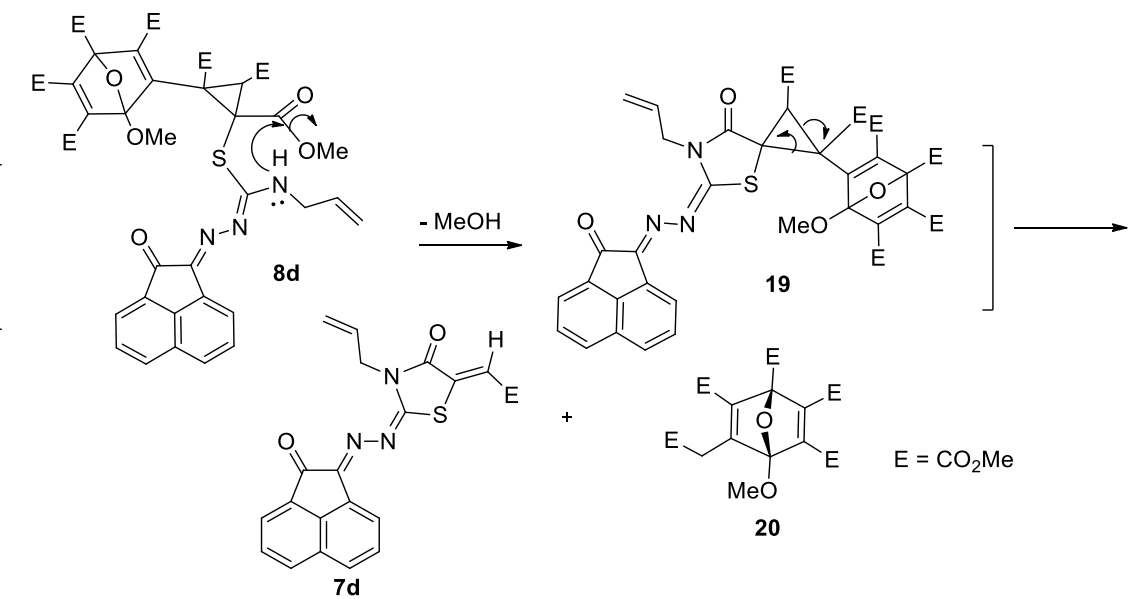

Scheme 6. Formation of thiazolidinone $7 \mathrm{~d}$ from compound $8 \mathrm{~d}$.

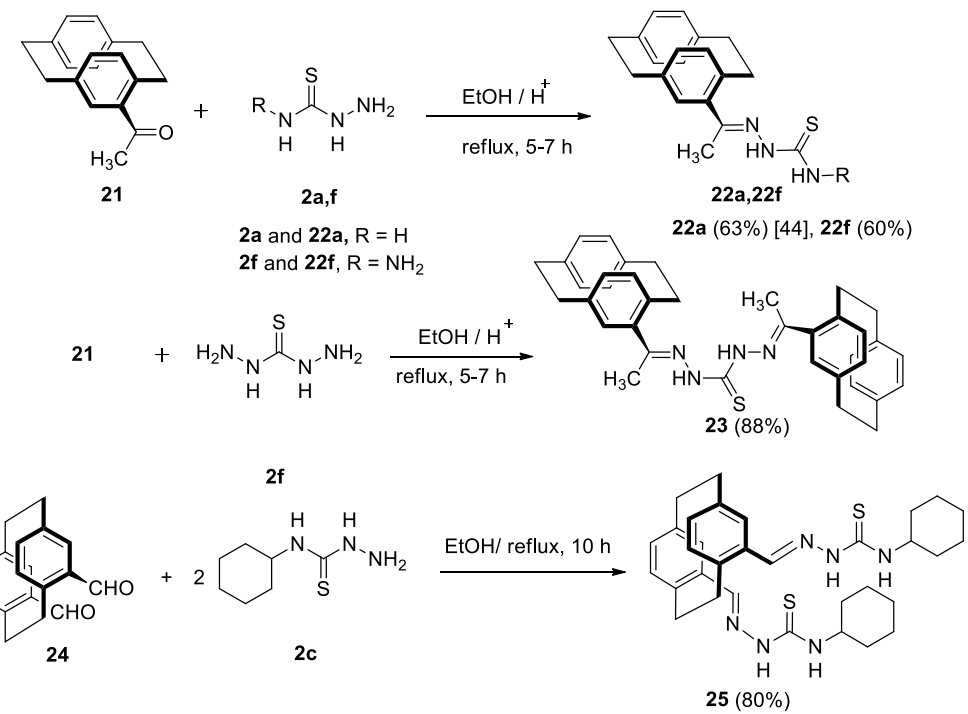

Scheme 7. Synthesis of paracyclophane thiosemicarbazone derivatives 22a,b, 23 and 25.

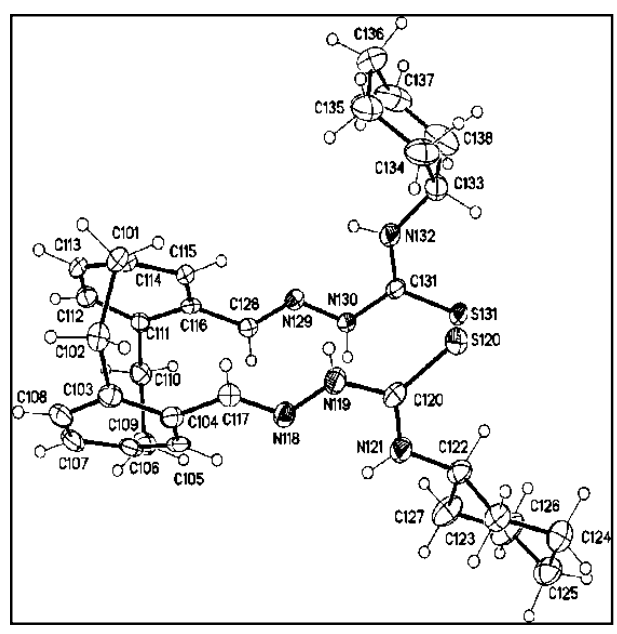

(a)

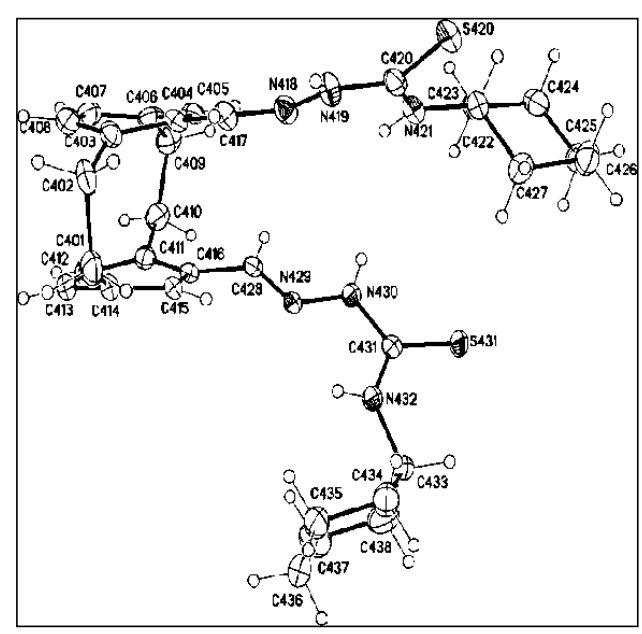

(b)

Figure 5. Molecular structure of two of the four crystallographic independent molecules of $\mathbf{2 5}$ showed the two different conformations in the crystal (displacement parameters, are drawn at $30 \%$ probability level). 
We aimed to synthesize thiazolidinones bearing a paracyclophanyl moiety; accordingly, we reacted 4-acetyl[2.2]paracyclophanylidene-thiosemicarbazone derivatives 22a and 22f with DMAD, $\mathbf{6 a}$ and DEAD, 6b (Scheme 8). By adaptation of the previously mentioned procedure, the expected 1,3-thiazolidinones 26-29 were obtained in good yields (Scheme 8). Depending on ${ }^{1} \mathrm{H}-\mathrm{NMR}$ and ${ }^{13} \mathrm{C}-\mathrm{NMR}$, various alternative structures were ruled out. In order to distinguish between the expected 1,3-thiazolidinone derivatives, a single crystal of 28 was obtained as ethyl 2-((Z)-2-(((E)-1-(1,4(1,4)-dibenzenacyclohexaphane-12-yl)ethylidene)hydrazine-ylidene)-4oxothiazolidin-5-yl)acetate (Figure 6).

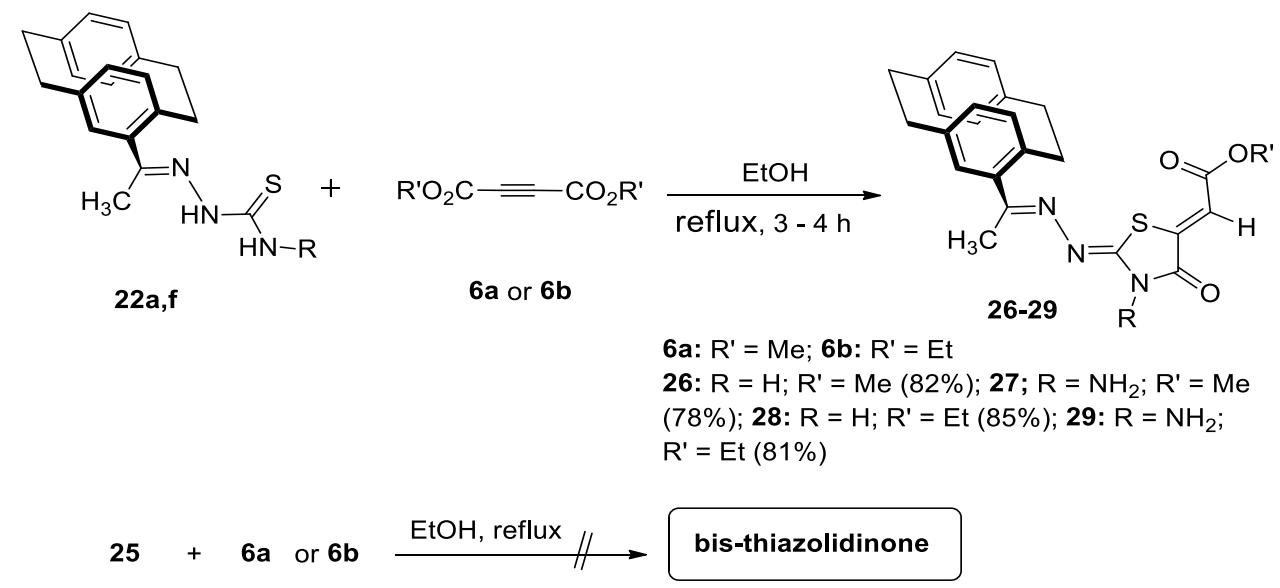

Scheme 8. Reaction of [2.2]paracyclophanylidene thiosemicarbazones $22 \mathbf{a}, \mathbf{f}$ with $\mathbf{6 a}$ and $\mathbf{6 b}$.

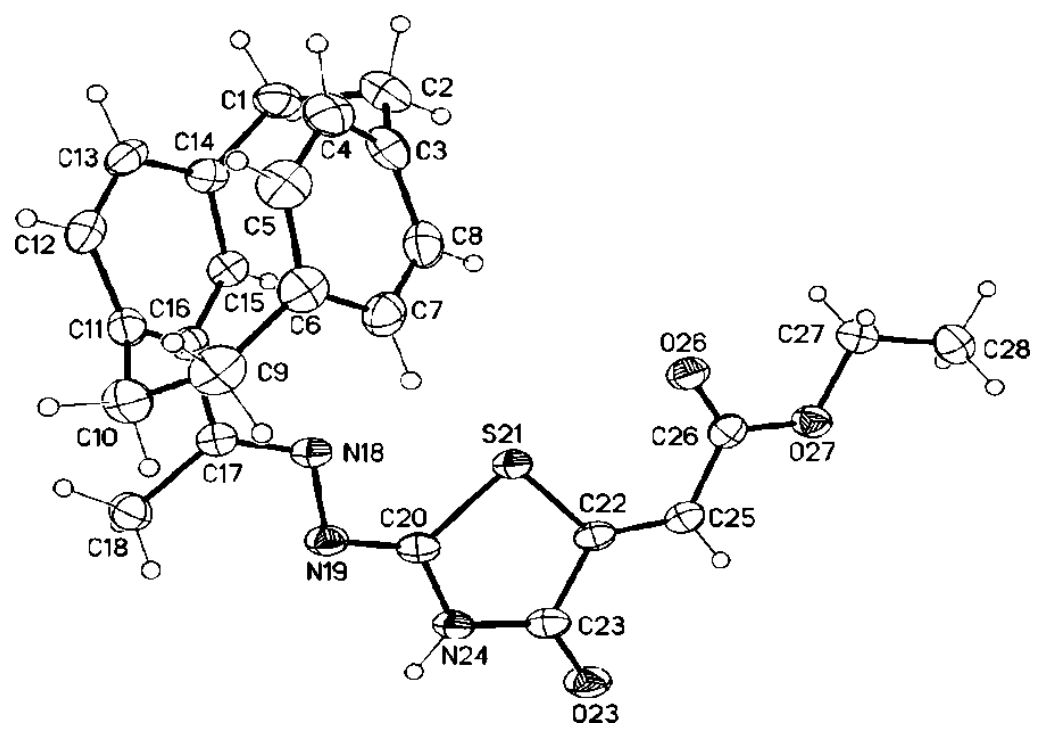

Figure 6. Molecular structure of $\mathbf{2 8}$ (displacement parameters are drawn at 30\% probability level).

Prolonged heating of $\mathbf{2 5}$ with either $\mathbf{6 a}$ or $\mathbf{6 b}$ under the aforementioned conditions failed to give either a mono- or a bis-thiazolidinone structure. Either the sensitivity of such layered molecules towards light and heat to undergo dimerization and/or steric factors might cause the reaction not to occur.

By analogy, the mechanism of formation of compounds 7a-e would be similar to that for the formation of compounds 26-29 (Scheme 9). Nucleophilic attack from sulfur lone pair in 22a,f to the triple bond of either $\mathbf{6 a}$ or $\mathbf{6 b}$ would give the intermediate $\mathbf{3 0}$, which would be neutralized as described before, to give 31 . The intermediate $\mathbf{3 1}$ would be easily cyclized to give the corresponding thiazolidinones 26-29 (Scheme 8), via another nucleophilic attack from $\mathrm{NH}$ in the formed intermediate 32 to the ester- $\mathrm{C}=\mathrm{O}$ and liberation of methanol or ethanol (Scheme 9). 


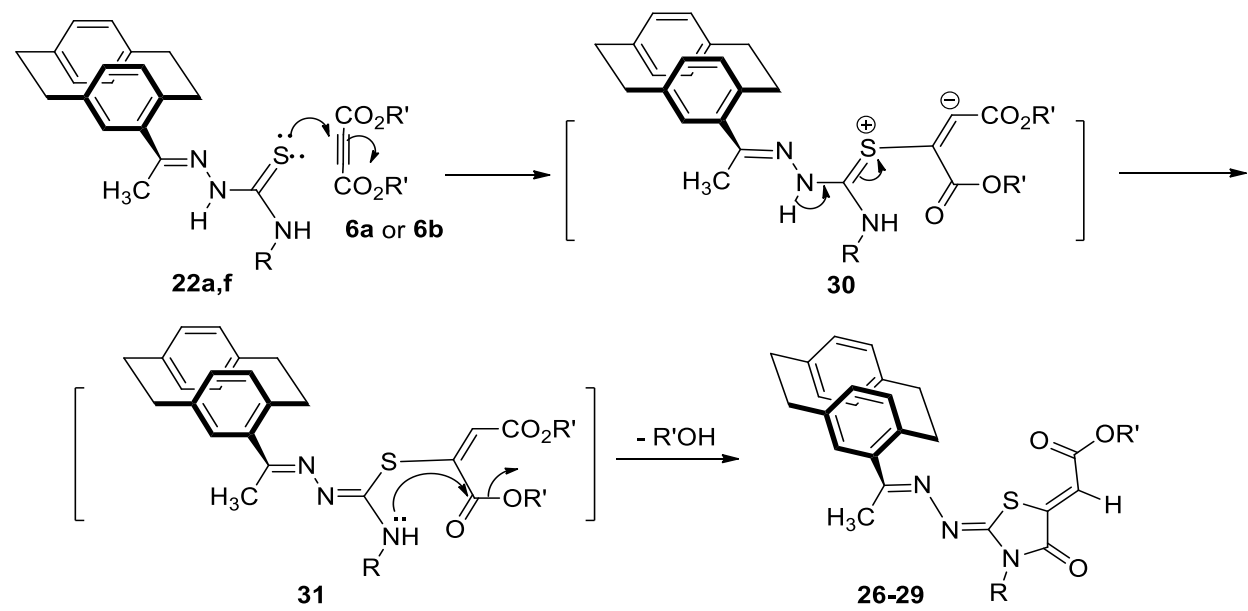

Scheme 9. Mechanism described the formation of compounds 26-29.

\section{Material and Methods}

\subsection{Chemistry}

Melting points (mp's) were recorded on a Gallenkamp melting point apparatus (Gallenkamp, UK) using open capillaries and were uncorrected. NMR data were recorded on Bruker AM 400 or AV400 spectrometers (Bruker, Rheinstetten, Germany), at $400 \mathrm{MHz}$ for ${ }^{1} \mathrm{H}$ and $100 \mathrm{MHz}$ for ${ }^{13} \mathrm{C}$. Chemical shifts were reported in ppm from tetramethylsilane using solvent resonance in $\mathrm{CDCl}_{3}$ or DMSO- $d_{6}$ solutions as the internal standard. The ${ }^{13} \mathrm{C}-\mathrm{NMR}$ signals were assigned on the basis of DEPT 135/90 spectra. The mass spectra were obtained on Finnigan MAT 312) (Germany) instrument using electron impact ionization $(70 \mathrm{eV})$. The IR spectra were recorded on Bruker Alpha FT-IR instrument (Germany) with samples prepared as potassium bromide pellets. Thin-layer chromatography (TLC) was performed on precoated plates (silica gel $60 \mathrm{PF}_{254}$ ), and zones were visualized with ultraviolet (UV) light. Elemental analyses for C, H, N were carried out with Elementar 306.

3.1.1. Starting Materials: Acenaphthequinone, 1 Was Bought and Bought from Aldrich, whereas [2.2]Paracyclophane Was Commercially Available

Preparation of 2-Oxoacenaphthylidene Thiosemicarbazones 5a-e

Compounds 5a-e were synthesized by refluxing solutions of acenaphthequinone $\mathbf{1}(1.82 \mathrm{~g}, 10$ $\mathrm{mmol})$ in absolute EtOH $(100 \mathrm{~mL})$ containing triethylamine $(0.5 \mathrm{~mL})$ and different solutions of thiosemicarbazide derivatives $\mathbf{2 a - e}$ for $3-5 \mathrm{~h}$. The products were left to stand, and then collected by filtration after precipitation with ethanol. The resulting solid was recrystallized from the stated solvents to give yellow to orange crystals.

(Z)-2-(2-Oxoacenaphthylen-1-(2H)-ylidene)hydrazinecarbothioamide (5a). Yield: (1.58 g, 70\%); orange crystals (EtOH); m.p.: $214-216{ }^{\circ} \mathrm{C}[50]$.

(Z)-2-(2-Oxoacenaphthylen-1-(2H)-ylidene)-N-phenylhydrazinecarbothioamide (5b). Yield: (2.65 g, 80\%); yellow crystals $(\mathrm{MeOH}) ;$ m.p.: $208-210{ }^{\circ} \mathrm{C}$ [47].

(Z)-N-Cyclohexyl-2-(2-oxoacenaphthylen-1-(2H)-ylidene)hydrazinecarbothioamide (5c). Yield: (2.80 g, 75\%); yellow crystals $\left(\mathrm{CH}_{3} \mathrm{CN}\right)$; m.p.: $202-204{ }^{\circ} \mathrm{C}$. IR (KBr): $v=3295\left(\mathrm{NH}^{\prime} \mathrm{s}\right), 2931$ (Ali-CH), 1681 (CO), 1620 $(\mathrm{C}=\mathrm{N}), 1609(\mathrm{Ar}-\mathrm{C}=\mathrm{C}) \mathrm{cm}^{-1} .{ }^{1} \mathrm{H}-\mathrm{NMR}\left(400 \mathrm{MHz}, \mathrm{DMSO}-d_{6}\right): \delta=1.17(\mathrm{~m}, 1 \mathrm{H}$, cyclic-CH) $1.33(\mathrm{~m}, 2 \mathrm{H}$, cyclic- $\left.\mathrm{CH}_{2}\right), 1.53\left(\mathrm{~m}, 2 \mathrm{H}\right.$, cyclic- $\left.\mathrm{CH}_{2}\right), 1.66(\mathrm{~m}, 1 \mathrm{H}$, cyclic- $\mathrm{CH}), 1.79\left(\mathrm{~m}, 2 \mathrm{H}\right.$, cyclic- $\left.\mathrm{CH}_{2}\right), 1.95(\mathrm{~m}, 2 \mathrm{H}$, cyclic- $\left.\mathrm{CH}_{2}\right), 4.23(\mathrm{~m}, 1 \mathrm{H}$, cyclic-CH), $7.85(t, 1 \mathrm{H}, \mathrm{Ar}-\mathrm{H}, J=7.9 \mathrm{~Hz}), 7.89(t, 1 \mathrm{H}, \mathrm{Ar}-\mathrm{H}, J=8.1 \mathrm{~Hz}), 8.09(d$, $1 \mathrm{H}, \mathrm{Ar}-\mathrm{H}, J=8.2 \mathrm{~Hz}), 8.10(d, 1 \mathrm{H}, \mathrm{Ar}-\mathrm{H}, J=8.2 \mathrm{~Hz}), 8.15(d, 1 \mathrm{H}, \mathrm{Ar}-\mathrm{H}, J=8.3 \mathrm{~Hz}), 8.38(\mathrm{~d}, 1 \mathrm{H}, \mathrm{Ar}-\mathrm{H}, J$ $=8.2 \mathrm{~Hz}), 8.97\left(d, 1 \mathrm{H}\right.$, cyclohexyl-NH), $12.62(\mathrm{~s}, 1 \mathrm{H},=\mathrm{N}-\mathrm{NH}) .{ }^{13} \mathrm{C}-\mathrm{NMR}:\left(100 \mathrm{MHz}\right.$, DMSO- $\left.d_{6}\right): \delta=$ 
24.87, 25.05, 31.44 (cyclic- $\left.\mathrm{CH}_{2}\right), 53.84$ (cyclic-CH), 118.61, 122.43, 127.05, 128.54, 128.79, 132.76 (Ar-CH), 129.88, 129.99, 130.43, 139.09 (Ar-C), $137.24(\mathrm{C}=\mathrm{N}), 176.04(\mathrm{C}=\mathrm{S}), 188.51(\mathrm{C}=\mathrm{O}) . \mathrm{MS}: \mathrm{m} / z(\%)=387\left(\mathrm{M}^{+}\right.$, 100), 180 (53), 154 (59), 136 (40), 107 (13). Anal. Calcd. for $\mathrm{C}_{19} \mathrm{H}_{19} \mathrm{~N}_{3} \mathrm{OS}$ (337.44); C, 67.63; $\mathrm{H}, 5.68 ; \mathrm{N}$, 12.45; S, 9.50. Found: C, 67.55; H, 5.75; N, 12.35; S, 9.57 .

(Z)-N-Allyl-2-(2-oxoacenaphthylen-1-(2H)-ylidene)hydrazinecarbothioamide (5d). Yield: (2.18 g, 74\%); light orange crystals (EtOH), m.p.: $190-191{ }^{\circ} \mathrm{C}$ [57].

(Z)-N-Ethyl-2-(2-oxoacenaphthylen-1(2H)-ylidene)hydrazinecarbothioamide (5e). Yield: (2.03 g, 72\%); yellow crystals (EtOH), m.p.: $195-196^{\circ} \mathrm{C}$; [47].

Reactions of 2-Oxoacenaphthylidene Thiosemicarbazone Derivatives with 6a

A mixture of 2-oxoacenaphthylidene thiosemicarbazones $(\mathbf{5 a}-\mathbf{e}, 1 \mathrm{mmol})$ in absolute ethanol $(50 \mathrm{~mL})$ was refluxed with $\mathbf{6 a}$ for $20-30 \mathrm{~min}$ and the reaction was monitored by TLC analysis. A yellow precipitate of $\mathbf{7 a - e}$ was formed, and then the reaction mixture was filtered and washed with a small amount of ethanol. The obtained precipitates were crystalized in ethanol to give yellow crystals of 1,3-thiazolidin-4-ones 7a-d. In addition, the filtrate was left to stand at room temperature after separation of the precipitates $\mathbf{7 a}-\mathbf{e}$ and, in the case of $\mathbf{7 d}$, fine crystals of $\mathbf{8 d}$ were collected after filtration).

(Z)-Methyl 2-((Z)-4-oxo-2-((E)-(2-oxoacenaphthylen-1-(2H)-ylidene)hydrazono)thiazolidin-5-ylidene)acetate (7a). Yield: (0.277 g, 76\%); yellow crystals (EtOH); m.p.: 252-254 ${ }^{\circ} \mathrm{C}$. IR (KBr): $v=3489(\mathrm{NH}), 3150$ (Ar-CH), $2960\left(\right.$ Ali-CH), 1715, $1696(\mathrm{CO}), 1623(\mathrm{C}=\mathrm{N}), 1604(\mathrm{Ar}-\mathrm{C}=\mathrm{C}) \mathrm{cm}^{-1}$. NMR see Table 1. MS, $\mathrm{m} / \mathrm{z}$ $(\%)=365\left(\mathrm{M}^{+}, 10\right), 307(35), 154(100), 137(65), 107$ (17). Anal. Calcd. for $\mathrm{C}_{18} \mathrm{H}_{11} \mathrm{~N}_{3} \mathrm{O}_{4} \mathrm{~S}$ (365.36); $\mathrm{C}$, 59.17; H, 3.03; N, 11.50; S, 8.78. Found: C, 59.26; H, 3.07; N, 11.57; S, 8.67.

(Z)-Methyl 2-((Z)-4-oxo-2-((E)-(2-oxoacenaphthylen-1-(2H)-ylidene)hydrazono)-3-phenyl-thiazolidin-5ylidene)acetate (7b). Yield: $(0.321 \mathrm{~g}, 73 \%)$; yellow crystals $(\mathrm{EtOH}) ; \mathrm{m} . \mathrm{p} .: 266-268^{\circ} \mathrm{C}$. IR $(\mathrm{KBr}): v=3120$ (Ar-CH), 2952 (Ali-CH), 1713, $1690(\mathrm{CO}), 1630(\mathrm{C}=\mathrm{N}), 1589(\mathrm{Ar}-\mathrm{C}=\mathrm{C}) \mathrm{cm}^{-1} .{ }^{1} \mathrm{H}-\mathrm{NMR}(400 \mathrm{MHz}$, DMSO- $\left.d_{6}\right): \delta=3.87\left(\mathrm{~s}, 3 \mathrm{H}, \mathrm{OCH}_{3}\right), 6.95(\mathrm{~s}, 1 \mathrm{H}$, vinyl-CH), $7.49(t, 1 \mathrm{H}, \mathrm{Ar}-\mathrm{H}, J=7.7 \mathrm{~Hz}), 7.66(\mathrm{~m}, 3 \mathrm{H}$, Ar-H), $7.69(\mathrm{~m}, 2 \mathrm{H}, \mathrm{Ar}-\mathrm{H}), 7.72(d, 1 \mathrm{H}, \mathrm{Ar}-\mathrm{H}, J=7.0 \mathrm{~Hz}), 7.86(t, 1 \mathrm{H}, \mathrm{Ar}-\mathrm{H}, J=8.0 \mathrm{~Hz}), 8.07(d, 1 \mathrm{H}$, Ar-H, $J=6.9 \mathrm{~Hz}), 8.13(d, 1 \mathrm{H}, \mathrm{Ar}-\mathrm{H}, J=8.3 \mathrm{~Hz}), 8.32(d, 1 \mathrm{H}, \mathrm{Ar}-\mathrm{H}, J=8.2 \mathrm{~Hz}) .{ }^{13} \mathrm{C}-\mathrm{NMR}(100 \mathrm{MHz}$, DMSO- $\left.d_{6}\right): \delta=52.72\left(\mathrm{OCH}_{3}\right), 116.42$ (vinyl-CH), 125.72, 127.19, 128.08, 128.21, 128.29, 128.74, 129.15, 129.20, 129.41 (Ar-CH), 130.06, 130.86, 132.14, 134.13, 140.69 (Ar-C), 140.77 (thiazole-C5), 155.55, 163.52 (thiazole-C2, $\mathrm{C}=\mathrm{N}), 165.77($ cyclic-C $=\mathrm{O}), 167.12($ ester $\mathrm{C}=\mathrm{O}), 188.41(\mathrm{C}=\mathrm{O}) . \mathrm{MS}, \mathrm{m} / \mathrm{z}(\%)=441\left(\mathrm{M}^{+}\right.$, 30), 306 (35), 180 (17), 154 (100), 136 (63), 107 (20). Anal. Calcd. for $\mathrm{C}_{24} \mathrm{H}_{15} \mathrm{~N}_{3} \mathrm{O}_{4} \mathrm{~S}$ (441.46); $\mathrm{C}, 65.30 ; \mathrm{H}$, 3.42; N, 9.52; S, 7.26. Found: C, 65.23; H, 3.38; N, 9.57; S, 7.37.

(Z)-Methyl 2-((Z)-3-cyclohexyl-4-oxo-2-((E)-(2-oxoacenaphthylen-1(2H)-ylidene)-hydrazono)-thiazolidin-5ylidene)acetate (7c). Yield: $(0.322 \mathrm{~g}$, $72 \%)$; yellow crystals $(\mathrm{EtOH}) ; \mathrm{m} . \mathrm{p} .: 284-286{ }^{\circ} \mathrm{C}$. IR $(\mathrm{KBr}): v=$ 3110 (Ar-CH), 2955 (Ali-CH), 1709, 1683 (CO), $1618(\mathrm{C}=\mathrm{N}), 1591(\mathrm{Ar}-\mathrm{C}=\mathrm{C}) \mathrm{cm}^{-1} .{ }^{1} \mathrm{H}-\mathrm{NMR}(400$ MHz, DMSO- $\left.d_{6}\right): \delta=1.35-1.37\left(\mathrm{~m}, 2 \mathrm{H}\right.$, cyclic- $\left.\mathrm{CH}_{2}\right), 1.72-1.75\left(\mathrm{~m}, 4 \mathrm{H}\right.$, cyclic- $\left.\mathrm{CH}_{2}\right), 1.86-1.88(\mathrm{~m}, 4 \mathrm{H}$, cyclic- $\left.\mathrm{CH}_{2}\right), 2.32(\mathrm{~m}, 1 \mathrm{H}$, cyclic- $\mathrm{CH}), 3.83\left(\mathrm{~s}, 3 \mathrm{H}, \mathrm{OCH}_{3}\right), 6.83(\mathrm{~s}, 1 \mathrm{H}$, vinyl-CH), $7.85(t, 1 \mathrm{H}, \mathrm{Ar}-\mathrm{H}, J=$ $8.0 \mathrm{~Hz}), 7.88(t, 1 \mathrm{H}, \mathrm{Ar}-\mathrm{H}, J=7.8 \mathrm{~Hz}), 7.93(d, 1 \mathrm{H}, \mathrm{Ar}-\mathrm{H}, J=7.1 \mathrm{~Hz}), 8.05(d, 1 \mathrm{H}, \mathrm{Ar}-\mathrm{H}, J=7.8 \mathrm{~Hz}), 8.13$ $(d, 1 \mathrm{H}, \mathrm{Ar}-\mathrm{H}, J=8.3 \mathrm{~Hz}), 8.26(d, 1 \mathrm{H}, \mathrm{Ar}-\mathrm{H}, J=8.3 \mathrm{~Hz}) .{ }^{13} \mathrm{C}-\mathrm{NMR}\left(100 \mathrm{MHz}, \mathrm{DMSO}-d_{6}\right): \delta=27.61$, 30.72, 35.93 (cyclic- $\left.\mathrm{CH}_{2}\right), 53.07\left(\mathrm{OCH}_{3}\right), 59.92$ (cyclic- $\left.\mathrm{CH}\right), 116.42$ (vinyl- $\left.\mathrm{CH}\right), 119.03,121.72,128.33$, 128.44, 128.92, 132.10 (Ar-CH), 131.23, 133.44, 131.93, 139.50 (Ar-C), 142.19 (thiazole-C5), 152.18, 155.56 (thiazole-C2, C=N), 166.37 (cyclic- $\mathrm{C}=\mathrm{O}), 166.69($ ester- $\mathrm{C}=\mathrm{O}), 183.87(\mathrm{C}=\mathrm{O}) . \mathrm{MS}, m / z(\%)=447\left(\mathrm{M}^{+}, 5\right)$, 306 (35), 154 (100) 137 (67), 107 (17). Anal. Calcd. for $\mathrm{C}_{24} \mathrm{H}_{21} \mathrm{~N}_{3} \mathrm{O}_{4} \mathrm{~S}$ (447.51); C, 64.41; H, 4.73; N, 9.39; S, 7.17. Found: $\mathrm{C}, 64.32 ; \mathrm{H}, 4.80 ; \mathrm{N}, 9.34 ; \mathrm{S}, 7.09$.

(Z)-Methyl 2-((Z)-3-allyl-4-oxo-2-((E)-(2-oxoacenaphthylen-1-(2H)-ylidene)hydrazono)-thiazolidin-5ylidene)acetate (7d). Yield: $(0.303 \mathrm{~g}, 75 \%)$; yellow crystals $(\mathrm{EtOH}) ; \mathrm{m} . \mathrm{p} .: 218-220^{\circ} \mathrm{C}$. IR $(\mathrm{KBr}): v=3085$ 
(Ar-CH), 2970 (Ali-CH), 1710, 1695 (CO), $1620(\mathrm{C}=\mathrm{N}), 1609$ (Ar-C=C) cm ${ }^{-1} .{ }^{1} \mathrm{H}-\mathrm{NMR}(400 \mathrm{MHz}$, DMSO- $\left.d_{6}\right): \delta=3.86\left(\mathrm{~s}, 3 \mathrm{H}, \mathrm{OCH}_{3}\right), 4.75\left(\mathrm{~m}, 2 \mathrm{H}\right.$, allyl- $\left.\mathrm{CH}_{2} \mathrm{~N}\right), 5.34\left(\mathrm{~m}, 2 \mathrm{H}\right.$, allyl- $\left.\mathrm{CH}_{2}=\right), 6.06(\mathrm{~m}, 1 \mathrm{H}$, allyl-CH=), $6.89(\mathrm{~s}, 1 \mathrm{H}$, vinyl-CH), $7.78(t, 1 \mathrm{H}, \mathrm{Ar}-\mathrm{H}, J=8.2 \mathrm{~Hz}), 7.81(t, 1 \mathrm{H}, \mathrm{Ar}-\mathrm{H}, J=7.9 \mathrm{~Hz}), 7.98(d$, $1 \mathrm{H}, \mathrm{Ar}-\mathrm{H}, J=7.1 \mathrm{~Hz}), 8.04(d, 1 \mathrm{H}, \mathrm{Ar}-\mathrm{H}, J=7.8 \mathrm{~Hz}), 8.12(d, 1 \mathrm{H}, \mathrm{Ar}-\mathrm{H}, J=8.1 \mathrm{~Hz}), 8.58(d, 1 \mathrm{H}, \mathrm{Ar}-\mathrm{H}, J=$ $8.1 \mathrm{~Hz}) .{ }^{13} \mathrm{C}-\mathrm{NMR}\left(100 \mathrm{MHz}, \mathrm{DMSO}-d_{6}\right): \delta=47.72\left(\right.$ allyl- $\left.\mathrm{CH}_{2} \mathrm{~N}\right) 52.61\left(\mathrm{OCH}_{3}\right), 115.28($ vinyl-CH$)$, 118.58 (allyl- $\mathrm{CH}_{2}=$ ), 118.90, 121.62, 128.35, 128.70, 129.06, 131.91 (Ar-CH), 131.21, 131.63, 132.40, 138.9 (Ar-C), 134.85 (allyl-CH=), 142.23 (thiazole-C5), 151.91, 155.36 (thiazole-C2, C = N) 165.41 (cyclic-C=O), 166.54 (ester $\mathrm{C}=\mathrm{O}), 183.93(\mathrm{C}=\mathrm{O})$. MS, $m / z(\%)=405\left(\mathrm{M}^{+}, 10\right), 306$ (40), 289 (16), 154 (100), 137 (65), 107 (15). Anal. Calcd. for $\mathrm{C}_{21} \mathrm{H}_{15} \mathrm{~N}_{3} \mathrm{O}_{4} \mathrm{~S}(405.43) ; \mathrm{C}, 62.21 ; \mathrm{H}, 3.73 ; \mathrm{N}, 10.36 ; \mathrm{S}, 7.91$. Found: $\mathrm{C}, 62.25 ; \mathrm{H}$, $3.68 ; \mathrm{N}, 10.45 ; \mathrm{S}, 7.97$.

(Z)-Methyl 2-((Z)-3-ethyl-4-oxo-2-((E)-(2-oxoacenaphthylen-1-(2H)-ylidene)hydrazono)-thiazolidin-5ylidene)acetate (7e). Yield: $(0.290 \mathrm{~g}, 74 \%)$; yellow crystals (EtOH); m.p.: $200-202{ }^{\circ} \mathrm{C}$. IR (KBr): $v=3143$ (Ar-CH), 2944 (Ali-CH), 1714, 1698 (CO), $1607(\mathrm{C}=\mathrm{N}), 1590(\mathrm{Ar}-\mathrm{C}=\mathrm{C}) \mathrm{cm}^{-1} .{ }^{1} \mathrm{H}-\mathrm{NMR}(400 \mathrm{MHz}$, DMSO- $\left.d_{6}\right): \delta=1.36\left(t, 3 \mathrm{H}, \mathrm{CH}_{3}, J=6.72 \mathrm{~Hz}\right), 3.75\left(q, 2 \mathrm{H}, \mathrm{CH}_{2}, J=6.72 \mathrm{~Hz}\right), 3.82\left(\mathrm{~s}, 3 \mathrm{H}, \mathrm{OCH}_{3}\right), 6.86$ $(\mathrm{s}, 1 \mathrm{H}$, vinyl-CH), $7.82(t, 1 \mathrm{H}, \mathrm{Ar}-\mathrm{H}, J=8.1 \mathrm{~Hz}), 8.01(d, 1 \mathrm{H}, \mathrm{Ar}-\mathrm{H}, J=7.2 \mathrm{~Hz}), 8.30(t, 1 \mathrm{H}, \mathrm{Ar}-\mathrm{H}, J=$ $7.9 \mathrm{~Hz}), 8.45(d, 1 \mathrm{H}, \mathrm{Ar}-\mathrm{H}, J=7.8 \mathrm{~Hz}), 8.65(d, 1 \mathrm{H}, \mathrm{Ar}-\mathrm{H}, J=8.2 \mathrm{~Hz}), 8.36(d, 1 \mathrm{H}, \mathrm{Ar}-\mathrm{H}, J=8.0 \mathrm{~Hz})$. ${ }^{13} \mathrm{C}-\mathrm{NMR}\left(100 \mathrm{MHz}, \mathrm{DMSO}-d_{6}\right): \delta=12.29\left(\mathrm{CH}_{3}\right), 31.5\left(\mathrm{CH}_{2}\right), 52.52\left(\mathrm{OCH}_{3}\right), 116.37$ (vinyl-CH), 126.02, 127.46, 128.85, 129.66, 130.03, 131.30 (Ar-CH), 132.37, 135.30, 139.91, 140.74 (Ar-C), 144.24 (thiazole-C5), 154.92, 160.66 (thiazole-C2, C=N), 164.13 (cyclic-C=O), 165.66 (ester $\mathrm{C}=\mathrm{O}), 187.49(\mathrm{C}=\mathrm{O}) . \mathrm{MS}, m / z(\%)=$ $393\left(\mathrm{M}^{+}, 20\right), 306(40), 153(100), 136(60), 107$ (17). Anal. Calcd. for $\mathrm{C}_{20} \mathrm{H}_{15} \mathrm{~N}_{3} \mathrm{O}_{4} \mathrm{~S}$ (393.42); $\mathrm{C}, 61.06 ; \mathrm{H}$, 3.84; N, 10.68; S, 8.15. Found: C, 61.16; H, 3.81; N, 10.75; S, 8.20.

Tetramethyl 5-(2-(((Z)-N-allyl-N'-((E)-2-oxoacenaphthylen-1-(2H)-ylidene)carbamo-hydrazonoyl)thio)-1,2,3tris(methoxycarbonyl)cyclopropyl)-4-methoxy-7-oxabicyclo-[2.2.1] hepta-2,5-diene-1,2,3,6-tetracarboxylate (8d). Yield: $(0.086 \mathrm{~g}, 10 \%)$; orange crystals (EtOH); m.p.: 224-226 ${ }^{\circ} \mathrm{C}$. IR $(\mathrm{KBr}): v=3401(\mathrm{NH}), 3100(\mathrm{Ar}-\mathrm{CH})$, 2953 (Ali-CH), 1717, 1698 (CO), $1623(\mathrm{C}=\mathrm{N}), 1590(\mathrm{Ar}-\mathrm{C}=\mathrm{C}) \mathrm{cm}^{-1} .{ }^{1} \mathrm{H}-\mathrm{NMR}\left(400 \mathrm{MHz}, \mathrm{DMSO}-d_{6}\right): \delta=$ $3.13\left(\mathrm{~s}, 1 \mathrm{H}\right.$, cyclopropane-H), $3.40\left(\mathrm{~s}, 3 \mathrm{H}, \mathrm{OCH}_{3}\right), 3.46,3.55,3.65,3.73,3.80,3.85,3.95\left(\mathrm{~s}, 3 \mathrm{H}, \mathrm{OCH}_{3}\right), 4.40$ $\left(\mathrm{m}, 2 \mathrm{H}\right.$, allyl- $\left.\mathrm{CH}_{2} \mathrm{~N}\right), 5.36\left(\mathrm{~m}, 2 \mathrm{H}\right.$, allyl- $\left.\mathrm{CH}_{2}=\right), 6.08(\mathrm{~m}, 1 \mathrm{H}$, allyl- $\mathrm{CH}=), 7.77(t, 1 \mathrm{H}, \mathrm{Ar}-\mathrm{H}, J=8.2 \mathrm{~Hz})$, $7.81(t, 1 \mathrm{H}, \mathrm{Ar}-\mathrm{H}, J=7.91 \mathrm{~Hz}), 8.00(d, 1 \mathrm{H}, \mathrm{Ar}-\mathrm{H}, J=7.1 \mathrm{~Hz}), 8.05(d, 1 \mathrm{H}, \mathrm{Ar}-\mathrm{H}, J=7.7 \mathrm{~Hz}), 8.11(d, 1 \mathrm{H}$, $\mathrm{Ar}-\mathrm{H}, J=8.3 \mathrm{~Hz}), 8.24(d, 1 \mathrm{H}, \mathrm{Ar}-\mathrm{H}, J=8.1 \mathrm{~Hz}), 9.40(\mathrm{~s}, \mathrm{IH}, \mathrm{NH}-\mathrm{allyl}) .{ }^{13} \mathrm{C}-\mathrm{NMR}\left(100 \mathrm{MHz}, \mathrm{DMSO}-d_{6}\right)$ : $\delta=22.23,24.90$ (cyclopropane-C), 25.60 (cyclopropane- $\mathrm{CH}), 46.81\left(\right.$ allyl- $\left.\mathrm{CH}_{2} \mathrm{~N}\right), 52.00,52.20,52.60$, 53.07, 53.19, 53.53, 53.65, $54.17\left(\mathrm{OCH}_{3}\right), 118.19$ (allyl- $\left.\mathrm{CH}_{2}=\right)$, 134.22 (allyl-CH=), 119.91, 121.80, 128.52, 128.29, 130.00, 132.32 (Ar-CH), 94.66, 121.66, 131.44, 134.58, 136.84, 138.01, 138.22, 138.93, 140.41, 142.82 (Ar-C), 152.63, $154.82(\mathrm{C}=\mathrm{N}), 165.21,167.53,168.91,171.68,172.00,173.51,175.44$ (ester-C=O), 185.16 $(\mathrm{C}=\mathrm{O})$. MS, $m / z(\%)=863\left(\mathrm{M}^{+}, 10\right), 805(5), 612(20), 307$ (35), 154 (100), 137 (67), 107 (18). Anal. Calcd. for $\mathrm{C}_{40} \mathrm{H}_{37} \mathrm{~N}_{3} \mathrm{O}_{17} \mathrm{~S}$ (863.80); C, 55.62; H, 4.32; N, 4.86; S, 3.71. Found: C, 55.69; H, 4.27; N, 4.79; S, 3.61 .

Preparation of 4-Acetyl-[2.2]Paracyclophanylidene-Thiosemicarbazones 22a, $\mathbf{f}$

4-Acetyl[2.2]paracyclophanylidene-thiosemicarbazones $\mathbf{2 2 a}, \mathbf{f}$ were synthesized via refluxing solutions of 4-acetyl[2.2]paracyclophane $(21)(2.50 \mathrm{~g}, 10 \mathrm{mmol})$ in absolute ethanol $(100 \mathrm{~mL})$ containing glacial acetic acid $(0.5 \mathrm{~mL})$ and thiosemicarbazide $2 \mathrm{a}(0.91 \mathrm{~g}, 10 \mathrm{mmol})$ or $(1.06 \mathrm{~g}$, $10 \mathrm{mmol}$ ) of thiocarbohydrazide $2 \mathrm{f}$ for $6 \mathrm{~h}$. Compounds 22a, $\mathbf{f}$ were precipitated, then filtered and washed with dry ethanol to get the crude as colorless crystals. On the other hand, Bis-4-acetyl[2.2]-paracyclophanylidene-hydrazine-1-carbothiohydrazide (23) was obtained efficiently after treating $21(2.50 \mathrm{~g}, 10 \mathrm{mmol})$ with $\mathbf{2 f}(0.53 \mathrm{~g}, 5 \mathrm{mmol})$ in absolute $\mathrm{EtOH}(50 \mathrm{~mL})$ under the same condition mentioned before (Scheme 7).

(E)-2-(4-([2.2]Paracyclophanylethylidene)-1-carbothiohydrazide (22f). Yield: $(2.03 \mathrm{~g}, 60 \%)$; colorless crystals (EtOH/DMF); m.p.: $188-189^{\circ} \mathrm{C} . \mathrm{IR}(\mathrm{KBr}): v=3450-3344(\mathrm{NH}), 2926-2853(\mathrm{Ali}-\mathrm{CH}), 1642(\mathrm{C}=\mathrm{N}), 1617$ $(\mathrm{Ar}-\mathrm{C}=\mathrm{C}), 1354(\mathrm{C}=\mathrm{S}) \mathrm{cm}^{-1}$. ${ }^{1} \mathrm{H}-\mathrm{NMR}\left(400 \mathrm{MHz}, \mathrm{DMSO}-d_{6}\right): \delta=2.28\left(\mathrm{~s}, 3 \mathrm{H}, \mathrm{CH}_{3}\right), 2.79(\mathrm{~m}, 2 \mathrm{H}$, PC-CH $\left.{ }_{2}\right), 2.98\left(\mathrm{~m}, 4 \mathrm{H}, 2 \mathrm{PC}-\mathrm{CH}_{2}\right), 3.40\left(\mathrm{~m}, 2 \mathrm{H}, \mathrm{PC}-\mathrm{CH}_{2}\right), 4.99$ (b, 2H, $\left.\mathrm{NH}_{2}\right), 6.49$ (m, 6H, PC-H), 6.86 (s, 
1H, PC-H), $9.37(\mathrm{~s}, 1 \mathrm{H}, \mathrm{NH}), 10.10(\mathrm{~s}, 1 \mathrm{H},=\mathrm{N}-\mathrm{NH}) .{ }^{13} \mathrm{C}-\mathrm{NMR}\left(100 \mathrm{MHz}, \mathrm{DMSO}-d_{6}\right): \delta=18.04\left(\mathrm{CH}_{3}\right)$, 34.38, 34.71, $34.84\left(\mathrm{Pc}_{-} \mathrm{CH}_{2}\right), 130.56,131.62,132.23,132.92,132.50,132.41,135.61$ (Pc-CH), 137.89, 138.97, 139.15, $139.45(\mathrm{Pc}-\mathrm{C}), 150.68(\mathrm{C}=\mathrm{N}), 177.17(\mathrm{C}=\mathrm{S}) . \mathrm{MS}: m / z(\%)=338\left[\mathrm{M}^{+}, 50\right], 306$ (30), 153 (100), 135 (75), 106 (26). Anal. Calcd. for $\mathrm{C}_{19} \mathrm{H}_{22} \mathrm{~N}_{4} \mathrm{~S}$ (338.47); $\mathrm{C}, 67.42 ; \mathrm{H}, 6.55 ; \mathrm{N}, 16.55 ; \mathrm{S}, 9.47$. Found: $\mathrm{C}, 67.46$; $\mathrm{H}, 6.50 ; \mathrm{N}, 16.49$; S, 9.53 .

Bis-4-acetyl[2.2]paracyclophanylidene-hydrazine-1-carbothiohydrazide (23). Yield: (4.14 g, 88\%); colorless crystals (EtOH/ DMF); m.p.: 224-226 ${ }^{\circ} \mathrm{C}$. IR (KBr): $v=3450-3344(\mathrm{NH}), 2926-2853$ (Ali-CH), 1642 $(\mathrm{C}=\mathrm{N}), 1617(\mathrm{Ar}-\mathrm{C}=\mathrm{C}), 1354(\mathrm{C}=\mathrm{S}) \mathrm{cm}^{-1} .{ }^{1} \mathrm{H}-\mathrm{NMR}\left(400 \mathrm{MHz}, \mathrm{DMSO}-d_{6}\right): \delta=2.29\left(\mathrm{~s}, 6 \mathrm{H}, 2 \mathrm{CH}_{3}\right), 2.35$ $\left(\mathrm{m}, 4 \mathrm{H}, \mathrm{PC}-\mathrm{CH}_{2}\right), 2.97\left(\mathrm{~m}, 8 \mathrm{H}, \mathrm{PC}-\mathrm{CH}_{2}\right), 3.10\left(\mathrm{~m}, 4 \mathrm{H}, \mathrm{PC}-\mathrm{CH}_{2}\right), 6.48-6.56(\mathrm{~m}, 4 \mathrm{H}, \mathrm{PC}-\mathrm{H}), 6.82(\mathrm{~s}, 2 \mathrm{H}$, PC-H), 7.08-7.09 (m, 8H, PC-H), 10.25 (s, 2H, 2=N-NH). ${ }^{13} \mathrm{C}-\mathrm{NMR}\left(100 \mathrm{MHz}\right.$, DMSO- $\left.d_{6}\right): \delta=18.56$, $18.69\left(\mathrm{CH}_{3}\right), 34.38,34.59,34.71,34.83\left(\mathrm{PC}^{-\mathrm{CH}_{2}}\right), 130.57,131.60,131.90,132.23,132.61,133.20,133.40$ (PC-CH), 135.61, 135.75, 138.23, 139.15, $139.24(\mathrm{PC}-\mathrm{C}), 150.93(\mathrm{C}=\mathrm{N}), 177.32(\mathrm{C}=\mathrm{S}) . \mathrm{MS}: \mathrm{m} / \mathrm{z}(\%)=570$ $\left[\mathrm{M}^{+}, 20\right], 363$ (15), 351 (25), 307 (34), 154 (100), 106 (20), 98 (63). Anal. Calcd. for $\mathrm{C}_{37} \mathrm{H}_{38} \mathrm{~N}_{4} \mathrm{~S}$ (570.79); $\mathrm{C}$, 77.86; H, 6.71; N, 9.82; S, 5.62. Found: C, 77.97; H, 6.63; N, 9.94; S, 5.75.

2,2'-(1,4(1,4)-Dibenzenacyclohexaphane-12 ${ }^{2} 4^{3}$-diylbis (methane-ylylidene))bis-(N-cyclohexyl-hydrazine-1carbothioamide) (25). Yield: $(4.59 \mathrm{~g}, 80 \%)$; colorless crystals $\left(\mathrm{CH}_{3} \mathrm{CN}\right)$; m.p.: $142-143^{\circ} \mathrm{C}$. IR $(\mathrm{KBr}): v=3440$ (NH), 2953 (Ali-CH), 1637 (C=N), 1610 (Ar-C=C), 1350 (C=S) cm ${ }^{-1} .{ }^{1} \mathrm{H}-\mathrm{NMR}\left(400 \mathrm{MHz}, \mathrm{DMSO}-d_{6}\right): \delta$ $=1.39\left(\mathrm{~m}, 4 \mathrm{H}\right.$, cyclohexyl- $\left.\mathrm{CH}_{2}\right), 1.75\left(\mathrm{~m}, 8 \mathrm{H}\right.$, cyclohexyl- $\left.\mathrm{CH}_{2}\right), 1.92\left(\mathrm{~m}, 8 \mathrm{H}\right.$, cyclohexyl $\left.-\mathrm{CH}_{2}\right), 2.55(\mathrm{~m}$, $2 \mathrm{H}$, cyclohexyl-CH), $2.90\left(\mathrm{~m}, 4 \mathrm{H}, 2 \mathrm{PC}-\mathrm{CH}_{2}\right), 3.23\left(\mathrm{~m}, 2 \mathrm{H}, \mathrm{PC}-\mathrm{CH}_{2}\right), 3.55\left(\mathrm{~m}, 2 \mathrm{H}, \mathrm{PC}-\mathrm{CH}_{2}\right), 6.52(\mathrm{~m}, 4 \mathrm{H}$, PC-H), 6.75 (s, 2H, PC-H), 7.55 (d, 2H, NH-cyclohexyl), $8.15(\mathrm{~s}, 2 \mathrm{H}, \mathrm{CH}=\mathrm{N}), 11.20(\mathrm{~s}, 2 \mathrm{H},=\mathrm{N}-\mathrm{NH})$. ${ }^{13} \mathrm{C}-\mathrm{NMR}\left(100 \mathrm{MHz}\right.$, DMSO- $\left.d_{6}\right): \delta=25.92,32.50,34.60\left(\right.$ cyclic- $\left.\mathrm{CH}_{2}\right), 32.58,34.65,34.88,\left(\mathrm{PC}^{-\mathrm{CH}_{2}}\right)$, 130.07, 132.40, 133.24 (PC-CH), 135.52, 138.14, 138.80, 139.90 (PC-C), $142.50(\mathrm{CH}=\mathrm{N}), 175.06$ (C = S). MS: $m / z(\%)=574\left[\mathrm{M}^{+}, 3\right], 417(4), 261$ (13), 258 (18), 157 (4), 141 (29), 129 (98), 83 (33), 56 (100). Anal. Calcd. for $\mathrm{C}_{32} \mathrm{H}_{42} \mathrm{~N}_{6} \mathrm{~S}_{2}$ (574.29); C, 66.86; H, 7.36; N, 14.62; S, 11.16. Found: C, 66.90; H, 7.42; N, 14.64; S, 11.10.

Reactions of 4-Acetyl[2.2]Paracyclophanylidene-Thiosemicarbazones, 22a, $\mathbf{f}$ with $\mathbf{6 a}$ and $\mathbf{6 b}$

A mixture of $\mathbf{2 2 a}, \mathbf{f}(1 \mathrm{mmol})$ in absolute ethanol $(50 \mathrm{~mL})$ was refluxed with either $\mathbf{6} \mathbf{a}$ or $\mathbf{6 b}$ for 3 $\mathrm{h}$ and the reaction was monitored by TLC. The precipitate of 26-29 was formed after concentration the solvent under vacuum, was filtered and washed with cold ethanol $(15 \mathrm{~mL})$ and recrystallized from methanol.

Methyl 2-((Z)-2-(((E)-1-(1,4(1,4)-dibenzenacyclohexaphane-12-yl)ethylidene)hydrazine-ylidene)-4oxothiazolidin-5-yl)acetate (26). Yield: $(0.355 \mathrm{~g}, 82 \%)$; yellow crystals; m.p.: $256-258{ }^{\circ} \mathrm{C}$. IR $(\mathrm{KBr}) v=3260(\mathrm{NH}), 3110(\mathrm{Ar}-\mathrm{CH}), 2925(\mathrm{Ali}-\mathrm{CH}), 1713,1699(\mathrm{CO}), 1626(\mathrm{C}=\mathrm{N}), 1600(\mathrm{Ar}-\mathrm{C}=\mathrm{C}) \mathrm{cm}^{-1}$. ${ }^{1} \mathrm{H}-\mathrm{NMR}\left(400 \mathrm{MHz}, \mathrm{DMSO}-d_{6}\right): \delta=2.37\left(\mathrm{~s}, 3 \mathrm{H}, \mathrm{CH}_{3}\right), 2.89\left(\mathrm{~m}, 8 \mathrm{H}, \mathrm{PC}-\mathrm{CH}_{2}\right), 3.77\left(\mathrm{~s}, 3 \mathrm{H}, \mathrm{OCH}_{3}\right), 6.54$ (s, 1H, PC-H), 6.68 (m, 6H, PC-H), 6.74 (s, 1H, vinyl-CH), 12.89 (s, 1H, NH-thiazole). ${ }^{13} \mathrm{C}-\mathrm{NMR}(100$ MHz, DMSO- $\left.d_{6}\right): \delta=18.33\left(\mathrm{CH}_{3}\right), 34.54,34.60,34.76,35.02\left(\mathrm{PC}^{\left.-\mathrm{CH}_{2}\right)}, 52.38\left(\mathrm{OCH}_{3}\right), 113.92(\right.$ vinyl-CH$)$, 130.81, 132.08, 132.37, 132.58, 132.70, 133.63, 135.82 (PC-CH), 138.23, 138.54, 138.97, 139.22 (PC-C), 143.54 (thiazole-C5), 159.05, 165.09 (thiazole-C2, C=N) 165.92 (cyclic-C=O), 165.99 (ester C=O). MS, $m / z(\%)=433\left(\mathrm{M}^{+}, 43\right), 306(40), 289(17), 153(100) 136(65), 106(15)$. Anal. Calcd. for $\mathrm{C}_{24} \mathrm{H}_{23} \mathrm{~N}_{3} \mathrm{O}_{3} \mathrm{~S}$ (433.52); C, 66.49; H, 5.35; N, 9.69; S, 7.40. Found: C, 66.52; H, 5.46; N, 9.57; S, 7.33.

Methyl 2-((Z)-3-amino-2-(((E)-1-(1,4(1,4)-dibenzenacyclohexaphane-12-yl)ethylidene)-hydrazineylidene)-4oxothiazolidin-5-yl)acetate (27). Yield: $\left(0.349 \mathrm{~g}\right.$, 78\%); yellow crystals $\left(\mathrm{CH}_{3} \mathrm{OH}\right) ;$ m.p.: $260-262{ }^{\circ} \mathrm{C}$. IR $(\mathrm{KBr}) v=3337\left(\mathrm{NH}_{2}\right), 3050(\mathrm{Ar}-\mathrm{CH}), 2923(\mathrm{Ali}-\mathrm{CH}), 1737,1700(\mathrm{CO}), 1630(\mathrm{C}=\mathrm{N}), 1603(\mathrm{Ar}-\mathrm{C}=\mathrm{C}) \mathrm{cm}^{-1}$. ${ }^{1} \mathrm{H}-\mathrm{NMR}\left(400 \mathrm{MHz}, \mathrm{DMSO}-d_{6}\right): \delta=2.35\left(\mathrm{~s}, 3 \mathrm{H}, \mathrm{CH}_{3}\right), 2.90\left(\mathrm{~m}, 8 \mathrm{H}, \mathrm{PC}^{-\mathrm{CH}_{2}}\right), 3.80\left(\mathrm{~s}, 3 \mathrm{H}, \mathrm{OCH}_{3}\right)$, $6.65(\mathrm{~m}, 6 \mathrm{H}, \mathrm{PC}-\mathrm{H}), 6.55(\mathrm{~s}, 1 \mathrm{H}, \mathrm{PC}-\mathrm{H}), 6.75\left(\mathrm{~s}, 1 \mathrm{H}\right.$, vinyl-CH), $6.90\left(\mathrm{br}, \mathrm{s}, 2 \mathrm{H}, \mathrm{NH}_{2}\right) .{ }^{13} \mathrm{C}-\mathrm{NMR}(100$ MHz, DMSO- $\left.d_{6}\right): \delta=18.61\left(\mathrm{CH}_{3}\right), 34.51,34.57,34.75,34.89\left(\mathrm{PC}^{\left.-\mathrm{CH}_{2}\right)}, 52.61\left(\mathrm{OCH}_{3}\right), 114.19(\right.$ vinyl-CH$)$, 130.48, 131.52, 132.24, 132.38, 132.58, 133.34, 135.68 (PC-CH), 138.11, 138.86, 139.97, 139.17 (PC-C), 144.51 (thiazole-C5), 152.36, 161.06 (thiazole-C2) 165.90 (cyclic-C=O), 166.77 (ester C = O). MS, $m / z(\%)$ 
$=448(\mathrm{M}+, 7), 306$ (30), 289 (15), 153 (100) 136 (64), 106 (20). Anal. Calcd. for $\mathrm{C}_{24} \mathrm{H}_{24} \mathrm{~N}_{4} \mathrm{O}_{3} \mathrm{~S}$ (448.54); C, 64.27; H, 5.39; N, 12.49; S, 7.15. Found: C, 64.39; H, 5.48; N, 12.37; S, 7.31.

Ethyl 2-((Z)-2-(((E)-1-(1,4(1,4)-dibenzenacyclohexaphane-12-yl)ethylidene) hydrazine-ylidene)-4-oxothiazolidin5-yl)acetate (28). Yield: $(0.380,85 \%)$; yellow plates $\left(\mathrm{CH}_{3} \mathrm{OH}\right)$; m.p. $200-202{ }^{\circ} \mathrm{C}$. IR $(\mathrm{KBr}) v=3240(\mathrm{NH})$, 3120 (Ar-CH), 2919 (Ali-CH), 1714, 1695 (CO), 1624 (C=N), 1592 (Ar-C=C) cm ${ }^{-1} .{ }^{1} \mathrm{H}-\mathrm{NMR}$ (DMSO- $d_{6}$ ): $\delta=1.27\left(t, 3 \mathrm{H}, \mathrm{CH}_{3}, J=7.1 \mathrm{~Hz}\right), 2.37\left(\mathrm{~s}, 3 \mathrm{H}, \mathrm{CH}_{3}\right), 2.90-3.14\left(\mathrm{~m}, 8 \mathrm{H}, \mathrm{PC}-\mathrm{CH}_{2}\right), 4.24\left(q, 2 \mathrm{H}, \mathrm{OCH}_{2}, J=\right.$ $7.1 \mathrm{~Hz}), 6.56-6.61$ (m, 6H, PC-H), 6.54 (s, 1H, PC-H), 6.70 (s, 1H, vinyl-CH), 12.90 (s, 1H, NH-thiazole). ${ }^{13} \mathrm{C}-\mathrm{NMR}\left(100 \mathrm{MHz}, \mathrm{DMSO}-d_{6}\right): \delta=14.01,18.36\left(\mathrm{CH}_{3}\right), 34.53,34.61,34.75,35.03\left(\mathrm{PC}^{-\mathrm{CH}_{2}}\right), 61.25$ $\left(\mathrm{OCH}_{2}\right), 114.56$ (vinyl-CH), 130.79, 132.09, 132.36, 132.58, 132.70, 133.70, 135.82 (PC-CH), 138.17, 138.55, 138.96, 139.22 (PC-C), 142.90 (thiazole-C5), 158.45, 165.27 (thiazole-C2, C=N), 165.32 (cyclic-C=O), 165.62 (ester $\mathrm{C}=\mathrm{O})$. MS, $m / z(\%)=447\left(\mathrm{M}^{+}, 62\right), 306$ (32), 153 (100), 135 (65), 106 (20). Anal. Calcd. For $\mathrm{C}_{25} \mathrm{H}_{25} \mathrm{~N}_{3} \mathrm{O}_{3} \mathrm{~S}$ (447.55): C, 67.09; H, 5.63; N, 9.39; S, 7.16. Found: $\mathrm{C}, 67.12 ; \mathrm{H}, 5.66 ; \mathrm{N}, 9.45 ; \mathrm{S}, 7.28$.

Ethyl 2-((Z)-3-Amino-2-(((E)-1-(1,4(1,4)-dibenzenacyclohexaphane-12-yl)ethylidene)-hydrazine-ylidene)-4oxothiazolidin-5-yl)acetate (29). Yield: $(0.374 \mathrm{~g}, 81 \%)$; yellow crystals $\left(\mathrm{CH}_{3} \mathrm{OH}\right) ;$ m.p. $174-175{ }^{\circ} \mathrm{C}$. IR (KBr): $v=3344(\mathrm{NH}), 3100(\mathrm{Ar}-\mathrm{CH}), 2924(\mathrm{Ali}-\mathrm{CH})$, 1735, $1720(\mathrm{CO}), 1622(\mathrm{C}=\mathrm{N}), 1607$ (Ar-C=C) $\mathrm{cm}^{-1} .{ }^{1} \mathrm{H}-\mathrm{NMR}\left(400 \mathrm{MHz}, \mathrm{DMSO}-d_{6}\right): \delta=1.28\left(t, 3 \mathrm{H}, \mathrm{CH}_{3}, J=7.2 \mathrm{~Hz}\right), 2.33\left(\mathrm{~s}, 3 \mathrm{H}, \mathrm{CH}_{3}\right), 2.92-3.04$ $\left(\mathrm{m}, 8 \mathrm{H}, \mathrm{PC}-\mathrm{CH}_{2}\right), 4.27\left(q, 2 \mathrm{H}, \mathrm{OCH}_{2}, J=7.2 \mathrm{~Hz}\right), 6.68(\mathrm{~m}, 6 \mathrm{H}, \mathrm{PC}-\mathrm{H}), 6.57(\mathrm{~s}, 1 \mathrm{H}, \mathrm{PC}-\mathrm{H}), 6.72(\mathrm{~s}, 1 \mathrm{H}$, vinyl-CH), 10.09 (b, s, 2H, NH 2$) .{ }^{13} \mathrm{C}-\mathrm{NMR}\left(100 \mathrm{MHz}, \mathrm{DMSO}-d_{6}\right): \delta=14.38\left(\mathrm{CH}_{3}\right), 18.62\left(\mathrm{CH}_{3}\right), 34.57$, 34.66, 34.76, 35.12, $\left(\mathrm{PC}-\mathrm{CH}_{2}\right), 54.40\left(\mathrm{OCH}_{3}\right), 62.0\left(\mathrm{OCH}_{2}\right), 115.46$ (vinyl-CH), 131.81, 132.18, 132.40, 132.60, 132.73, 135.72, 134.86 (PC-CH), 138.27, 138.60, 138.99, 139.25 (PC-C), 143.56 (thiazole-C5), 159.17, 166.02 (thiazole-C2, C=N), 165.95 (cyclic-C=O), 176.02 (ester $\mathrm{C}=\mathrm{O}) . \mathrm{MS}, m / z(\%)=462\left(\mathrm{M}^{+}, 6\right), 307(25)$, 248 (40), 155 (27), 154 (95), 143 (100), 135 (65), 106 (20). Anal. Calcd. for $\mathrm{C}_{25} \mathrm{H}_{26} \mathrm{~N}_{4} \mathrm{O}_{3} \mathrm{~S}$ (462.56): $\mathrm{C}$, 64.91; H, 5.67; N, 12.11; S, 6.93. Found: C, 65.01; H, 5.75; N, 12.16; S, 7.02.

\subsection{Single Crystal X-ray Structure Determination of $\mathbf{5 a}, \mathbf{7 d}, \mathbf{8 d}, \mathbf{2 5}$ and $\mathbf{2 8}$}

A single crystal of 5 a was obtained by recrystallization from $\mathrm{C}_{2} \mathrm{H}_{5} \mathrm{OH}$ (ethanol), a single crystal of $\mathbf{7 d}$ was obtained by recrystallization from $\mathrm{C}_{2} \mathrm{H}_{5} \mathrm{OH}$ (ethanol), a single crystal of $\mathbf{8} \mathbf{d}$ was obtained from $\mathrm{C}_{2} \mathrm{H}_{5} \mathrm{OH}$ (ethanol), 25 from $\mathrm{CH}_{3} \mathrm{CN}$ (acetonitrile), and a single crystal of 28 was obtained acenaphthequinone recrystallization from $\mathrm{CH}_{3} \mathrm{OH}$ (methanol). The single-crystal X-ray analyses were carried out on a Bruker D8 Venture diffractometer with Photon100 or Photon II CPAD detector at 123K using Cu-K $\alpha$ radiation $(\lambda=1.54178 \AA$ ). Direct Methods (SHELXS [58] or Dual Space Methods (SHELXT [59]) were used for structure solution and refinement was carried out using SHELXL-2014 (full-matrix least-squares on $F^{2}$ ) [59]. Hydrogen atoms were refined using a riding model $(\mathrm{H}(\mathrm{N}, \mathrm{O})$ free). Semi-empirical absorption corrections were applied.

Compound 5a (SB1184_HY): $\mathrm{C}_{13} \mathrm{H}_{9} \mathrm{~N}_{3} \mathrm{OS}, \mathrm{Mr}=255.29 \mathrm{~g} \mathrm{~mol}^{-1}$, orange blocks, size $0.30 \times 0.25 \times$ $0.20 \mathrm{~mm}$, monoclinic, space group P2 $1 / \mathrm{c}$ (no.14), $a=6.3357$ (2) $\AA$, $b=18.2558$ (7) $\AA$, $c=20.0737$ (7) $\AA \hat{\Lambda}, \beta=97.327(1)^{\circ}, \mathrm{V}=2302.83(14) \AA^{3}, \mathrm{Z}=8, \mathrm{D}_{\text {calcd }}=1.473 \mathrm{Mg} \mathrm{m}^{-3}, \mathrm{~F}(000)=1056, \mu(\mathrm{Cu}-\mathrm{K} \alpha)=$ $2.42 \mathrm{~mm}^{-1}, \mathrm{~T}=123 \mathrm{~K}, 19236$ measured reflections $\left(2 \theta_{\max }=144.2^{\circ}\right), 4510$ independent reflections $\left[R_{\text {int }}=0.023\right]$, 343 parameters, 6 restraints, $R_{1}[$ for $4468 \mathrm{I}>2 \sigma(\mathrm{I})]=0.030, w \mathrm{R}^{2}$ (for all data) $=$ $0.079, \mathrm{~S}=1.06$, largest diff. peak and hole $=0.37 \mathrm{e} \AA^{-3} /-0.31$ e $\AA^{-3}$. It is a polymorph of EBINOV (2-(2-oxoacenaphthylen-1(2H)-ylidene)hydrazinecarbothioamide methanol solvate) [60].

Compound 7d (SB1186_HY): $\mathrm{C}_{21} \mathrm{H}_{15} \mathrm{~N}_{3} \mathrm{O}_{4} \mathrm{~S}, \mathrm{Mr}=405.42 \mathrm{~g} \mathrm{~mol}^{-1}$, yellow blocks, size $0.12 \times 0.06 \times$ $0.04 \mathrm{~mm}$, monoclinic, space group P2 $1 / \mathrm{n}$ (no.14), $a=7.0903$ (2) $\AA$, $b=15.6914$ (5) $\AA$, $c=16.4943$ (5) $\AA$, $\beta$ $=98.917(1)^{\circ}, \mathrm{V}=1812.92(9) \AA^{3}, \mathrm{Z}=4, \mathrm{D}_{\text {calcd }}=1.485 \mathrm{Mg} \mathrm{m}^{-3}, \mathrm{~F}(000)=840, \mu(\mathrm{Cu}-\mathrm{K} \alpha)=1.90 \mathrm{~mm}^{-1}, \mathrm{~T}$ $=123 \mathrm{~K}, 18041$ measured reflections $\left(2 \theta_{\max }=144.4^{\circ}\right), 3575$ independent reflections $\left[R_{\text {int }}=0.024\right], 263$ parameters, $\mathrm{R}_{1}$ [for $\left.3402 \mathrm{I}>2 \sigma(\mathrm{I})\right]=0.029, \mathrm{w} R^{2}$ (for all data) $=0.077, \mathrm{~S}=1.06$, largest diff. peak and hole $=0.28$ e $\AA^{-3} /-0.30$ e $\AA^{-3}$. 
Compound 8d (SB1185_HY): $\mathrm{C}_{40} \mathrm{H}_{37} \mathrm{~N}_{3} \mathrm{O}_{17} \mathrm{~S}, \mathrm{Mr}=863.78 \mathrm{~g} \mathrm{~mol}^{-1}$, orange plates, size $0.09 \times 0.06 \times$ $0.03 \mathrm{~mm}$, triclinic, space group $P-1$ (no. 2), $a=12.8863$ (5) $\AA$, $b=15.1023$ (6) $\AA$, $c=22.3027$ (8) $\AA$, $\alpha$ $=100.800(2)^{\circ}, \beta=100.850(2)^{\circ}, \gamma=104.460(2)^{\circ}, \mathrm{V}=4000.4(3) \AA^{3}, \mathrm{Z}=4, \mathrm{D}_{\text {calcd }}=1.434 \mathrm{Mg} \mathrm{m}^{-3}, \mathrm{~F}$ $(000)=1800, \mu(\mathrm{Cu}-\mathrm{K} \alpha)=1.43 \mathrm{~mm}^{-1}, \mathrm{~T}=123 \mathrm{~K}, 71781$ measured reflections $\left(2 \theta_{\max }=144.4^{\circ}\right), 15705$ independent reflections $\left[R_{\text {int }}=0.039\right], 1113$ parameters, 986 restraints, $R_{1}$ [for 14,020 $\left.\mathrm{I}>2 \sigma(\mathrm{I})\right]=0.059$, $\mathrm{w} R^{2}$ (for all data) $=0.148, \mathrm{~S}=1.10$, largest diff. peak and hole $=1.52 \mathrm{e} \AA^{-3} /-0.59 \mathrm{e} \AA^{-3}$. In the 2nd molecule the vinyl moiety is disordered, disordered atoms refined isotropically. In the second molecule the oxacenaphthylene moiety shows high Uij-values, probably disordered, but the disorder is not resolved. Use of constraints (EADP) and restraints (SADI) for the refinement as well as a general RIGU restraint.

Compound 25 (SB1014_HY): $\mathrm{C}_{32} \mathrm{H}_{42} \mathrm{~N}_{6} \mathrm{~S}_{2} \cdot 0.5 \mathrm{C}_{2} \mathrm{H}_{5} \mathrm{~N}, \mathrm{Mr}=595.36 \mathrm{~g} \mathrm{~mol}^{-1}$, colorless blocks, size 0.40 $\times 0.18 \times 0.06 \mathrm{~mm}$, triclinic, space group $P-1$ (no.2), $a=13.7588$ (6) $\AA$, $b=23.3483$ (9) $\AA$, $c=23.3963$ (9) $\AA \hat{,}, \alpha=60.101(2)^{\circ}, \beta=84.894(3)^{\circ}, \gamma=84.516(3)^{\circ}, \mathrm{V}=6478.3(5) \AA^{3}, \mathrm{Z}=8, \mathrm{D}_{\text {calcd }}=1.221 \mathrm{Mg} \mathrm{m}^{-3}, \mathrm{~F}$ $(000)=2552, \mu(\mathrm{Cu}-\mathrm{K} \alpha)=1.74 \mathrm{~mm}^{-1}, \mathrm{~T}=123 \mathrm{~K}, 67389$ measured reflections $\left(2 \theta_{\max }=145.4^{\circ}\right), 24776$ independent reflections [ $R_{\text {int }}=0.067$ ], 1485 parameters, 1254 restraints, $R_{1}$ [for $\left.16,926 \mathrm{I}>2 \sigma(I)\right]=0.115$, $\mathrm{w} R^{2}$ (for all data) $=0.321, \mathrm{~S}=1.01$, largest diff. peak and hole $=1.33 \mathrm{e} \AA^{-3} /-0.79 \mathrm{e} \AA^{-3}$. The compound was refined as a two-component pseudo merohedral twin. A general RIGU restraint is used for the refinement due to the bad quality of the data. Two solvent molecules acetonitrile per asymmetric unit disordered, the disordered atoms refined isotropically.

Compound 28 (SB1183_HY): $\mathrm{C}_{25} \mathrm{H}_{25} \mathrm{~N}_{3} \mathrm{O}_{3} \mathrm{~S} \cdot \mathrm{CH}_{3} \mathrm{OH}, \mathrm{Mr}=479.58 \mathrm{~g} \mathrm{~mol}^{-1}$, yellow plates, size $0.16 \times$ $0.14 \times 0.02 \mathrm{~mm}$, monoclinic, space group $P 2_{1} / \mathrm{c}(\mathrm{no}, .14), a=19.5995$ (5) $\AA$, $b=7.4181$ (2) $\AA$, $c=18.2170$ (5) $\AA$, $\beta=112.833(2)^{\circ}, \mathrm{V}=2441.04(12) \AA^{3}, \mathrm{Z}=4, \mathrm{D}_{\text {calcd }}=1.305 \mathrm{Mg} \mathrm{m}^{-3}, \mathrm{~F}(000)=1016, \mu(\mathrm{Cu}-\mathrm{K} \alpha)=$ $1.49 \mathrm{~mm}^{-1}, \mathrm{~T}=123 \mathrm{~K}, 25915$ measured reflections $\left(2 \theta_{\max }=144.6^{\circ}\right), 4808$ independent reflections $\left[R_{\text {int }}\right.$ $=0.052], 315$ parameters, two restraints, $R_{1}[$ for $4146 \mathrm{I}>2 \sigma(\mathrm{I})]=0.064, \mathrm{w} R^{2}$ (for all data) $=0.155, \mathrm{~S}=$ 1.17, largest diff. peak and hole $=0.46 \mathrm{e} \AA^{-3} /-0.32 \mathrm{e} \AA^{-3}$.

CCDC 1937480 (5a-sb1184_hy), 1937481 (7d-sb1186_hy), 1937482 (8d-sb1185_hy), 1937483 (25-sb1014_hy), and 1937484 (28-sb1183_hy) contain the Supplementary crystallographic data for this paper. These data can be obtained free of charge from The Cambridge Crystallographic Data Centre via www.ccdc.cam.ac.uk/data_request/cif (details in Supplementary Materials).

\section{Conclusions}

In conclusion, we prepared various derivatives of thiazolodinone derived by acenaphthequinone and [2.2]paracyclophane. We are able here to prove the structures of the obtained products via X-ray structure analysis. We could also, for the first time, get the X-ray structure analysis of the tetramer form resulting from DMAD.

Supplementary Materials: The following are available online.

Author Contributions: Writing-A.A.A.; revision-N.K.M., A.A.H., K.M.E.-S.; experimental work-M.M.M.; revision and submission-S.B.; X-ray-M.N.; NMR-A.B.B.

Funding: The authors thank the Science and Technology Development Fund, STDF, Egypt for its financial supporting of the Grant No. 22934. However this research received no other external funding. The NMR spectrometer at Florida Institute of Technology was purchased with the assistance of the U.S. National Science Foundation (CHE 03 42251).

Conflicts of Interest: The authors declare no conflict of interest. 


\section{References}

1. Suthar, S.K.; Jaiswal, V.; Lohan, S.; Bansal, S.; Chaudhary, A.; Tiwari, A.; Alex, A.T.; Joseph, A. Novel quinolone substituted thiazolidin-4-ones as anti-inflammatory, anticancer agents: Design, synthesis and biological screening. Eur. J. Med. Chem. 2013, 63, 589-602. [CrossRef] [PubMed]

2. Aly, A.A.; Ishak, E.A.; El Malah, T.; Brown, A.B.; Elayat, W.M. Synthesis of potentially antioxidant and antibacterial biologically active thiazolidines. J. Heterocycl. Chem. 2015, 52, 1758-1764. [CrossRef]

3. Sharma, S.; Sharma, P.K.; Kumar, N.; Dudhe, R. A review on various heterocyclic moieties and their antitubercular activity. Biomed. Pharmacother. 2011, 65, 244-251. [CrossRef] [PubMed]

4. Patel, D.; Kumari, P.; Patel, N. Synthesis and biological evaluation of some thiazolidinones as antimicrobial agents. Eur. J. Med. Chem. 2012, 48, 354-362. [CrossRef] [PubMed]

5. Rawal, R.K.; Tripathi, R.; Katti, S.B.; Pannecouque, C.; de Clercq, E. Design, synthesis, and evaluation of 2-aryl-3-heteroaryl-1,3-thiazolidin-4-ones as anti-HIV agents. Bioorg. Med. Chem. 2007, 15, 1725-1731. [CrossRef] [PubMed]

6. Shingalapur, R.V.; Hosamani, K.M.; Keri, R.S.; Hugar, M.H. Derivatives of benzimidazole pharmacophore: Synthesis, anticonvulsant, antidiabetic and DNA cleavage studies. Eur. J. Med. Chem. 2010, 45, 1753-1759. [CrossRef] [PubMed]

7. Kishore, A.; Nampurath, G.K.; Mathew, S.P.; Zachariah, R.T.; Potu, B.K.; Rao, M.S.; Valiathan, M.; Chamallamudi, M.R. Antidiabetic effect through islet cell protection in streptozotocin diabetes: A preliminary assessment of two thiazolidin-4-ones in Swiss albino mice. Chem. Biol. Interact. 2009, 177, 242-246. [CrossRef]

8. Aly, A.A.; Brown, A.B.; El-Emary, T.I.; Ewas, A.M.M.; Ramadan, M. Hydrazinecarbothioamide group in the synthesis of heterocycles. Arkivoc 2009, 2009, 150-197.

9. Hassan, A.A.; Mohamed, N.K.; Makhlouf, M.M.; Bräse, S.; Nieger, M. Reactions of dimethyl acetylenedicarboxylate with 2,5-dithiobiurea derivatives. Synthesis 2014, 46, 3097-3102. [CrossRef]

10. Hassan, A.A.; Mohamed, N.K.; Makhlouf, M.M.; Bräse, S.; Nieger, M.; Höpf, H. (Hex-2-en-ylidene)- $N$-substituted hydrazonecarbothioamides and 2,3-dichloro-1,4-naphthoquinone: Nucleophilic substitution reactions and synthesis of naphtho[2,3-f][1,3,4]-triazepines and naphtho[2,3-d] thiazoles. Synthesis 2016, 48, 3134-3140. [CrossRef]

11. Aly, A.A.; Brown, A.B.; Abdel-Aziz, M.; Abuo-Rahma, G.E.D.A.; Radwan, M.F.; Ramadan, M.; Gamal-Eldeen, A.M. Synthesis of new 4-oxo-thiazolidine-5-ylidenes of antitumor and antioxidant activities. J. Heterocycl. Chem. 2010, 47, 547-554. [CrossRef]

12. Aly, A.A.; Brown, A.B.; Abdel-Aziz, M.; Abuo-Rahma, G.E.D.A.; Radwan, M.F.; Ramadan, M.; Gamal-Eldeen, A.M. An efficient synthesis of thiazolidin-4-ones with antitumor and antioxidant activities. J. Heterocycl. Chem. 2012, 49, 726-731. [CrossRef]

13. Hassan, A.A.; Abdel-Latif, F.F.; Nour El-Din, A.M.; Mostafa, S.M.; Nieger, M.; Bräse, S. Synthesis of (E)-2,5-disubstituted 1,3,4-thiadiazolyl-2,3-diphenylpropenones from alkenylidene-hydrazinecarbothioamides. Tetrahedron 2012, 68, 8487-8492. [CrossRef]

14. Hu, W.-X.; Zhou, W.; Xia, C.-N.; Wen, X. Synthesis and anticancer activity of thiosemicarbazones. Bioorg. Med. Chem. Lett. 2006, 16, 2213-2218. [CrossRef] [PubMed]

15. Barbuceanu, S.-F.; Ilies, D.C.; Saramet, G.; Uivarosi, V.; Draghici, C.; Radulescu, V. Synthesis and antioxidant activity evaluation of new compounds from hydrazinecarbothioamide and 1,2,4-triazole class containing diarylsulfone and 2,4-difluorophenyl moieties. Int. J. Mol. Sci. 2014, 15, 10908-10925. [CrossRef] [PubMed]

16. Paiva, R.O.; Kneipp, L.F.; Goular, C.M.; Albuquerque, M.A.; Echevarria, A. Antifungal activities of thiosemicarbazones and semicarbazones against mycotoxigenic fungi. Ciência Agrotecnol. 2014, 38, 531-537. [CrossRef]

17. Reis, D.C.; Despaigne, A.A.R.; Da Silva, J.G.; Silva, N.F.; Vilela, C.F.; Mendes, I.C.; Takahashi, J.A.; Beraldo, H. Structural studies and investigation on the activity of imidazole-derived thiosemicarbazones and hydrazones against crop-related fungi. Molecules 2013, 18, 12645-12662. [CrossRef]

18. El Ashry, E.S.H.; Abdel Hamid, H.; Kassem, A.A.; Shoukry, M. Synthesis and reactions of acenaphthenequinones. Part 2. The reactions of acenaphthenequinones. Molecules 2002, 7, 155-188. [CrossRef] 
19. Mhaidat, I.; Mergos, J.A.; Hamilakis, S.; Kollia, C.; Loizos, Z.; Tsolomitis, A.; Dervos, C.T. Synthesis and reactions of acenaphthenequinones. Part 2. The reactions of acenaphthenequinones. Mater. Lett. 2009, 63, 2587-2590. [CrossRef]

20. Ziarani, G.M.; Hajiabbasi, P.; Gholamzadeh, P. Development of the acenaphthenequinone reactions. Heterocycles 2012, 85, 1869-1890. [CrossRef]

21. Yavari, I.; Khajeh-Khezri, A. Recent advances in the synthesis of hetero- and carbocyclic compounds and complexes based on acenaphthylene-1,2-dione. Synthesis 2018, 50, 3947-3973. [CrossRef]

22. Hyatt, J.L.; Wadkins, R.M.; Tsurkan, L.; Hicks, L.D.; Hatfield, M.J.; Edwards, C.C.; Ross, C.R., II; Cantalupo, S.A.; Crundwell, G.; Danks, M.K.; et al. Planarity and constrain of the carbonyl groups in 1,2-diones are determinants for selective inhibition of human carboxylesterase 1. J. Med. Chem. 2007, 50, 5727-5734. [CrossRef] [PubMed]

23. Rodriguez-Argüelles, M.C.; Ferrari, M.B.; Fava, G.G.; Pelizzi, C.; Pelosi, G.; Albertini, R.; Bonati, A.; Dall'Aglio, P.P.; Lunghi, P.; Pinelli, S. Acenaphthenequinone thiosemicarbazone and its transition metal complexes: Synthesis, structure, and biological activity. J. Inorg. Biochem. 1997, 66, 7-17. [CrossRef]

24. Zhang, Z.; Yang, H.; Wu, G.; Li, Z.; Song, T.; Li, X.Q. Probing the difference between BH3 groove of Mcl-1 and Bcl-2 protein: Implications for dual inhibitors design. Eur. J. Med. Chem. 2011, 46, 3909-3916. [CrossRef] [PubMed]

25. El-Alawi, Y.S.; McConkey, B.J.; Dixon, D.G.; Greenberg, B.M. Measurement of short- and long-term toxicity of polycyclic aromatic hydrocarbons using luminescent bacteria. Ecotoxicol. Environ. Saf. 2002, 51, $12-21$. [CrossRef] [PubMed]

26. Nair, V.; Biju, A.T.; Vinod, A.U.; Suresh, E. Reaction of Huisgen Zwitter ion with 1,2-benzoquinones and isatins: Synthesis of dihydro-1,2,3-benzoxadiazoles and spirooxadiazolines. Org. Lett. 2005, 7, 5139-5142. [CrossRef] [PubMed]

27. Wang, C.; Jiang, Y.-H.; Yan, C-G. Convenient synthesis of spiro (indoline-3,4'-pyrano[2,3-c]pyrazole) and spiro (acenaphthyl-3,4'-pyrano[2,3-c]pyrazoles via four-component reaction. Chin. Chem. Lett. 2015, 26, 889-893. [CrossRef]

28. Yavari, I.; Baoosi, L.; Halvagar, M.R. A synthesis of fused acenaphthopyrrolizines via the 1,3-dipolar cycloaddition reaction of azomethine ylides with acetylenic esters. Mol. Divers. 2017, 21, 257-263. [CrossRef]

29. Wei, A.C.; Ali, M.A.; Yoon, Y.K.; Ismail, R.; Choon, T.S.; Kumar, R.S. A facile three-component [3+2]-cycloaddition for the regioselective synthesis of highly functionalized dispiropyrrolidines acting as antimycobacterial agents. Bioorg. Med. Chem. Lett. 2013, 23, 1383-1386. [CrossRef]

30. Arumugam, N.; Almansour, A.I.; Kumar, R.S.; Perumal, S.; Ghabbour, H.A.; Fun, H.-K. A 1,3-dippolar cycloaddition-annulation protocol for the expedient region-, stereo-, and product-selective construction of novel hybrid heterocycles comprising seven rings and seven contiguous stereocentres. Tetrahedron Lett. 2013, 54, 2515-2519. [CrossRef]

31. Song, L.-L.; Yang, C.; Yu, Y.-Q.; Xu, D.-Z. A simple and green tandem Knoevenagel-phospha-Michael reaction for one-pot synthesis of 2-oxindol-3-yl-phosphonates catalyzed by a DABCO-based ionic liquid. Synthesis 2017, 49, 1641-1647.

32. Yavari, I.; Baoosi, L.; Halvagar, M.R. A convenient synthesis of fused tetrahydroazocines from acenaphthylene-1,2-dione, proline, and acetylenic esters. Synlett 2018, 29, 635-639. [CrossRef]

33. Wang, X.-H.; Yan, C.-G. Facile synthesis of spiro(indane-2,1'-pyrrolo[2,1-a] isoquinolines) via three-component reaction of isoquinolinium salts, indane-1,3-dione, and isatins. Synthesis 2014, 46, 1059-1066. [CrossRef]

34. Gong, H.; Sun, J.; Yan, C.-G. Efficient synthesis of polycyclic dispirooxindoles via domino Diels-Alder cyclodimerization reaction. Tetrahedron 2014, 70, 6641-6650. [CrossRef]

35. Ahadi, S.; Hosseini, G.; Bazgir, A. Synthesis of oxo-indolin-3-ylidene-1,3-dithioles. J. Iran. Chem. Soc. 2012, 9, 333-338. [CrossRef]

36. Kiruthika, S.E.; Lakshmi, N.V.; Banu, B.R.; Perumal, P.T. A facile strategy for the one pot multicomponent synthesis of spiro dihydropyridines from amines and activated alkynes. Tetrahedron Lett. 2011, 52, 6508-6511. [CrossRef]

37. Hopf, H. [2.2] Paracyclophanes in polymer chemistry and materials science. Angew. Chem. Int. Ed. 2008, 47, 9808-9812. [CrossRef] [PubMed]

38. Gibson, S.E.; Knight, J.D. [2.2] Paracyclophane derivatives in asymmetric catalysis. Org. Biomol. Chem. 2003, 1, 1256-1269. [CrossRef] [PubMed] 
39. Gulder, T.; Baran, P.S. Strained cyclophane natural products: Macrocyclization at its limits. Nat. Prod. Rep. 2012, 29, 899-934. [CrossRef] [PubMed]

40. Hassan, Z.; Spuling, E.; Knoll, D.M.; Bräse, S. Regioselective functionalization of [2.2] paracyclophanes: Recent synthetic progress and perspectives. Angew. Chem. Int. Ed. 2019, in press. [CrossRef] [PubMed]

41. Aly, A.A.; Brown, A.B. Asymmetric and fused heterocycles based on [2.2] paracyclophane. Tetrahedron 2009, 65, 8055-8089. [CrossRef]

42. Aly, A.A.; Hopf, H.; Ernst, L.; Dix, I.; Jones, P.G. New cycloadditions of $(E)-N, \alpha$-dimethyl- $\alpha-(4-[2.2]$ paracyclophanyl)nitrone. Eur. J. Org. Chem. 2006, 2006, 3001-3006. [CrossRef]

43. Aly, A.A. Cycloaddition of (E)-N-\{2-([2.2]paracyclophan-4-yl)ethylidene\}methylamine $N$-oxide with 2,3-diphenylcyclopropenones and dibenzoyl acetylene: Synthesis of new paracyclophanylpyrroles. J. Chem. Res. 2007, 2007, 451-454. [CrossRef]

44. Aly, A.A.; Hopf, H.; Jones, P.G.; Dix, I. Cycloadditions of $\alpha$-(4-[2.2]paracyclophane)- $N$-methyl nitrone. Tetrahedron 2006, 62, 4498-4505. [CrossRef]

45. Hopf, H.; Aly, A.A.; Swaminathan, V.N.; Ernst, L.; Dix, I.; Jones, P.G. A simple route to a pyridinyl[2.2]paracyclophane. Eur. J. Org. Chem. 2005, 2005, 68-71. [CrossRef]

46. Aly, A.A.; Bräse, S.; Weis, P. Tridentate and bidentate copper complexes of [2.2] paracyclophanyl-substituted thiosemicarbazones, thiocarbazones, hydrazones, and thioureas. J. Mol. Struct. 2019, 1178, 311-326. [CrossRef]

47. Pascu, S.L.; Waghorn, P.A.; Churchill, G.C.; Sim, R.B. Synthesis of Metal Complexes with Thiosemicarbazone Derivatives for Use in Medical Imaging and Therapy. PCT International Application WO 2008025941 A2 20080306, 4 August 2008.

48. Patil, P.B.; Patil, J.D.; Korade, S.N.; Kshirsagar, S.D.; Govindwar, S.P.; Pore, D.M. An efficient synthesis of anti-microbial 1,2,4-triazole-3-thiones promoted by acidic ionic liquid. Res. Chem. Intermed. 2016, 42, 4171-4180. [CrossRef]

49. Mohammadi, M.K.; Firuzi, O.; Khoshneviszadeh, M.; Razzaghi-Asl, N.; Sepehri, S.; Miri, R. Novel 9-(alkylthio)-acenaphtho[1,2-e]-1,2,4-triazine derivatives: Synthesis, cytotoxic activity, and molecular docking studies on B-cell lymphoma 2 (Bcl-2). DARU J. Pharm. Sci. 2014, 22, 2. [CrossRef] [PubMed]

50. Satheshkumar, A.; El-Mossalamy, E.H.; Manivannam, R.; Parthiban, C.; Al-Harbi, L.M.; Kosa, S.; Elango, K.P. Anion induced azo-hydrazine tautomerism for the selective colorimetric sensing of fluoride ion. Spectrochim. Acta A 2014, 128, 798-805. [CrossRef] [PubMed]

51. Kauer, J.C.; Simmons, H.E. Tetramers of acetylenedicarboxylic esters. J. Org. Chem. 1968, 33, $2720-2726$. [CrossRef]

52. Hocking, M.B.; van der Voort Maarschalk, F.W. X-ray structures of triphenylphosphine and 1,3,5-triphenylphosphole products with dimethyl acetylenedicarboxylate tetramer. Can. J. Chem. 1994, 72, 2428-2442. [CrossRef]

53. Winterfeldt, E.; Giesler, G. Formation of trimethyl 2-methoxyfurantricarboxylate from dimethyl acetylenedicarboxylate. Angew. Chem. Int. Ed. 1966, 5, 579. [CrossRef]

54. Banert, K.; Bochmann, S.; Ihle, A.; Plefka, O.; Taubert, F.; Walther, T.; Korb, M.; Rueffer, T.; Lang, H. Synthesis with perfect atom economy: Generation of furan derivatives by 1,3-dipolar cycloaddition of acetylenedicarboxylates at cyclooctynes. Molecules 2014, 19, 14022-14035. [CrossRef] [PubMed]

55. Medina, S.; Gonzalez-Gomez, A.; Dominguez, G.; Perez-Castells, J. Medium-sized and strained heterocycles from non-catalyzed and gold-catalyzed conversions of $\beta$-carbolines. Org. Biomol. Chem. 2012, 10, 7167-7176. [CrossRef] [PubMed]

56. Huang, H.; Luo, H.; Tao, G.; Cai, W.; Cao, J.; Duan, Z.; Mathey, F. Selective synthesis of (Z)-diazadiphosphafulvalene from 2,2'-bis-azaphosphindole. Org. Lett. 2018, 20, 1027-1030. [CrossRef] [PubMed]

57. Arrowsmith, R.L.; Waghorn, P.A.; Jones, M.W.; Bauman, A.; Brayshaw, S.K.; Hu, Z.; Kociok-Köhn, G.; Mindt, T.L.; Tyrrell, R.M.; Botchway, S.W.; et al. Fluorescent gallium and indium bis(thiosemicarbazonates) and their radiolabeled analogues: Synthesis, structures, and cellular confocal imaging investigations. Dalton Trans. 2011, 40, 6238-6252. [CrossRef] [PubMed]

58. Sheldrick, G.M. A short history of SHELX. Acta Crystallogr. 2008, 64, 112-122. [CrossRef] 
59. Sheldrick, G.M. SHELXT-Integrated space-group and crystal-structure determination. Acta Crystallogr. 2015, 71, 3-8. [CrossRef]

60. Tadjarodi, A.; Najjari, S.; Notash, B. Synthesis and crystal structure of a new thiosemicarbazone, acenaphthenequinone thiosemiscarbazone mono methanol. Iran. J. Crystallogr. Miner. 2015, 22, 109-114.

Sample Availability: Samples of the compounds are not available from the authors.

(C) 2019 by the authors. Licensee MDPI, Basel, Switzerland. This article is an open access article distributed under the terms and conditions of the Creative Commons Attribution (CC BY) license (http://creativecommons.org/licenses/by/4.0/). 